\title{
European Stroke Organisation (ESO)-European Society for Minimally Invasive Neurological Therapy (ESMINT) expedited recommendation on indication for intravenous thrombolysis before mechanical thrombectomy in patients with acute ischemic stroke and anterior circulation large vessel occlusion
}

\author{
Guillaume Turc (D) , 'Georgios Tsivgoulis (D) , 2,3 Heinrich J Audebert, ${ }^{4}$ \\ Hieronymus Boogaarts (D), ${ }^{5}$ Pervinder Bhogal (D) , ${ }^{6}$ Gian Marco De Marchis (D) , \\ Ana Catarina Fonseca (1) ${ }^{8}$ Pooja Khatri (1) , Mikaël Mazighi (1) , ${ }^{9}, 11$ \\ Natalia Pérez de la Ossa, ${ }^{12}$ Peter D Schellinger, ${ }^{13}$ Daniel Strbian, ${ }^{14}$ Danilo Toni, ${ }^{15}$ \\ Philip White (D) ${ }^{16}$ William Whiteley (D) , ${ }^{17}$ Andrea Zini (D) , ${ }^{18}$ Wim van Zwam (D) , 19 \\ Jens Fiehler (iD 20
}

\begin{abstract}
- Additional supplemental material is published online only. To view, please visit the journal online (http://dx.doi. org/10.1136/neurintsurg-
\end{abstract} 2021-018589).

For numbered affiliations see end of article.

\section{Correspondence to} Professor Guillaume Turc, Neurology, GHU Paris Pôle Neuro Sainte-Anne, Paris, Île-de-France, France; g.turc@ ghu-paris.fr

This article is being co-published in the following journals: European Stroke Journal and Journal of Neurolnterventional Surgery in 2022.

Received 20 December 2021 Accepted 9 January 2022
Check for updates

(c) Author(s) (or their employer(s)) 2022. No commercial re-use. See rights and permissions. Published by BMJ.

To cite: Turc G, Tsivgoulis $\mathrm{G}$, Audebert $\mathrm{HJ}$, et al.

J Neurolntervent Surg Epub ahead of print: [please include Day Month Year]. doi:10.1136/

neurintsurg-2021-018589

\section{ABSTRACT}

Six randomized controlled clinical trials have assessed whether mechanical thrombectomy (MT) alone is noninferior to intravenous thrombolysis (IVT) plus MT within 4.5hours of symptom onset in patients with anterior circulation large vessel occlusion (LVO) ischemic stroke and no contraindication to IVT. An expedited recommendation process was initiated by the European Stroke Organisation (ESO) and conducted with the European Society of Minimally Invasive Neurological Therapy (ESMINT) according to ESO standard operating procedure based on the GRADE system. We identified two relevant Population, Intervention, Comparator, Outcome (PICO) questions, performed systematic reviews and meta-analyses of the literature, assessed the quality of the available evidence, and wrote evidence-based recommendations. Expert opinion was provided if insufficient evidence was available to provide recommendations based on the GRADE approach.

For stroke patients with anterior circulation LVO directly admitted to a MT-capable center ('mothership') within 4.5 hours of symptom onset and eligible for both treatments, we recommend IVT plus MT over MT alone (moderate evidence, strong recommendation). MT should not prevent the initiation of IVT, nor should IVT delay MT. In stroke patients with anterior circulation LVO admitted to a center without MT facilities and eligible for IVT $\leq 4.5$ hours and MT, we recommend IVT followed by rapid transfer to a MT capable-center ('drip-and-ship') in preference to omitting IVT (low evidence, strong recommendation). Expert consensus statements on ischemic stroke on awakening from sleep are also provided. Patients with anterior circulation LVO stroke should receive IVT in addition to MT if they have no contraindications to either treatment.

\section{INTRODUCTION}

Intravenous thrombolysis (IVT) with alteplase was the first acute ischemic stroke reperfusion therapy proven to be effective, initially within 3 hours and later within 4.5 hours, ${ }^{2}$ and was more recently proven for patients with ischemic stroke on awakening. ${ }^{3}$ This evidence led to the evolution of an effective infrastructure for acute stroke care in Europe and elsewhere. On this established infrastructural backbone, mechanical thrombectomy (MT) showed encouraging results in single arm studies and subsequently was proven to have a major therapeutic effect in several randomized controlled clinical trials (RCTs), demonstrating the superiority of MT combined with IVT ('bridging therapy') over IVT alone in patients with a large vessel occlusion (LVO). Among patients with acute ischemic stroke and no contraindications to IVT, bridging therapy is the standard treatment for LVO within the first 4.5 hours after symptom onset. ${ }^{5}$

With accumulating experience and the proven effectiveness of MT even in patients with IVT contraindications, it was highly relevant to assess whether MT as a stand-alone therapy would be sufficient even in patients eligible for IVT. Within the last few months, four RCTs have been published comparing MT alone (direct MT) with bridging therapy. ${ }^{6-9}$ An early meta-analysis suggested that MT alone may be non-inferior to MT plus IVT with alteplase, but this conclusion was not based on stringent non-inferiority margins and only included data from the first three RCTs. ${ }^{10}$ After the recent presentation of the results of the SWIFT DIRECT and DIRECT-SAFE trials and without further RCTs on this topic to be presented in the near future, ${ }^{11} 12$ the time appeared appropriate for the European Stroke Organisation (ESO) and the European Society of Minimally Invasive Neurological Therapy (ESMINT) to coordinate and publish an expedited recommendation on the role of IVT before MT.

\section{METHODS}

This joint expedited recommendation was initiated by the ESO and prepared according to the ESO 
standard operating procedure, ${ }^{1314}$ which is based on the Grading of Recommendations, Assessment, Development and Evaluations (GRADE) system. ${ }^{15}$ The ESO and ESMINT Guideline Boards and Executive Committees reviewed the intellectual and financial disclosures of all module working group (MWG) members (online supplemental table 1) and approved the composition of the group, which was chaired by Guillaume Turc and Jens Fiehler.

The steps undertaken by the MWG are summarized as follows:

1. The single topic of whether IVT should be administered before MT in patients with LVO acute ischemic stroke was considered in this expedited recommendation. However, the MWG deemed it to be important to consider separately the role of IVT in those patients directly admitted to a thrombectomy-capable center ('mothership') versus those admitted to a stroke unit without thrombectomy facilities ('drip-and-ship' paradigm); this is because the average time between the start of IVT infusion and arterial puncture differs markedly between these two treatment paradigms.

2. A list of relevant outcomes was produced and rated by each MWG member using secret ballot voting on a scale from 1 (not important) to 9 (extremely important). The mean value for each outcome is reported below. According to GRADE, five outcomes were considered to be of critical importance (mean score of 7-9).

- Good (clinical) outcome, ${ }^{16}$ defined as 90-day modified Rankin Scale (mRS) scores 0-2: 8.8

- Reduced disability ${ }^{16}$ ( $\geq 1$ point reduction across all mRS scores at 90 days): 7.7

- Excellent outcome, ${ }^{16}$ defined as 90 -day mRS scores 0-1: 7.6

- Symptomatic intracranial hemorrhage (sICH): 7.2

- Mortality at 90 days: 7.0

- Successful reperfusion (modified Treatment In Cerebral Ischemia (mTICI) score $\geq 2 \mathrm{~b}$ ) at the end of the endovascular procedure: 6.4

- Time between symptom onset and successful reperfusion: 5.5

- Time between door and successful reperfusion: 5.3

- Distal embolization or embolization in a new territory: 5.3

- First-pass complete reperfusion: 5.1

- Time between arterial puncture and reperfusion: 5.1

- Time between symptom onset and arterial puncture: 4.9

- Time between door and arterial puncture: 4.9

- Infarct volume at 24-36 hours defined by expert neuroradiologists: 4.9

- Any ICH: 4.6

- Number of passes: 3.8

Based on this expert vote, good outcome (mRS 0-2 at 90 days) was defined as the outcome of highest priority and was considered first. Unless specified otherwise, reduced disability ${ }^{16}$ corresponded to a reduction of at least one point in the mRS score at 90 days across all mRS grades ('shift analysis'). sICH was defined according to each study's original criterion. In the case of limited data for the outcomes of highest importance, outcomes of lesser importance were also considered.

3. The MWG formulated a list of Population, Intervention, Comparator, Outcome (PICO) questions, which were reviewed and subsequently approved by external reviewers and members of the ESO and ESMINT Guidelines Boards and Executive Committees.
4. The recommendation for the first PICO question was based on a systematic review of RCTs of direct MT versus bridging therapy. To this aim, we have updated the results of a previously published systematic review that was conducted up to January $2021 .^{10}$ We have applied the same search strategy for a period from January 2021 to December 2021. We have also included results of RCTs presented at international conferences but which were not published at the time of preparation of this document. For the second PICO question, no RCT was available. We have therefore included non-randomized studies identified in a recent systematic review in our quantitative synthesis. ${ }^{17}$

5. The risk of bias in each RCT was assessed using the Cochrane Collaboration's Risk of Bias 2 (RoB2) tool. ${ }^{18}$

6. Whenever appropriate, random-effects meta-analyses were conducted using Stata software version 16.0 (Statacorp). Results were summarized as odds ratios (ORs) or common ORs (cORs) with 95\% confidence interval (95\% CI). For mRS-related outcomes, risk ratios (RRs) were used as summary measures in sensitivity analyses. Heterogeneity across studies was assessed using the $\mathrm{I}^{2}$ statistic. Heterogeneity was classified as low $\left(\mathrm{I}^{2}<30 \%\right)$, moderate $\left(\mathrm{I}^{2} \geq 30 \%\right)$, substantial $\left(\mathrm{I}^{2} \geq 50 \%\right)$, or considerable $\left(\mathrm{I}^{2} \geq 75 \%\right)$.

7. Before statistical analyses were conducted the MWG decided that the assessment of non-inferiority would be based on the absolute difference ('risk difference' (RD)) in the proportions of patients achieving good outcome between the two treatment groups (MT alone vs bridging therapy, the latter being the reference group). A non-inferiority margin was chosen via secret ballot voting. The minimal and maximal values for a non-inferiority margin advocated by MWG members were $1 \%$ and $5 \%$, respectively. A majority (10/18) of MWG members voted for a margin of $1.3 \%$, which corresponds to the median minimal clinically important difference in a survey of US stroke neurologists. ${ }^{19}$ Therefore, we prespecified that for the present expedited recommendation, non-inferiority would be met if the lower 95\% CI boundary of the random-effects pooled RD was superior or equal to $-1.3 \%$. For the main analysis, random-effects pooled RD was calculated using the DerSimonian and Laird method. ${ }^{20}$ We also conducted two sensitivity analyses to calculate the pooled RD. The first one was based on (1) the random-effects pooled proportion of patients with good outcome in the bridging therapy arm, and (2) the randomeffects pooled RR $(95 \% \mathrm{CI})$ for good outcome. The second sensitivity analysis corresponded to the calculation of a fixed-effect pooled RD (inverse variance method). No $p$ value for non-inferiority was computed.

8. The results of data analyses were imported into the GRADEpro Guideline Development Tool (McMaster University, 2015; developed by Evidence Prime, Inc). For each PICO question and each outcome, the risk of bias was assessed and the quality of evidence was rated as high, moderate, low or very low based on the type of available evidence (randomized or observational studies) and considerations on inconsistency of results, indirectness of evidence, imprecision of results, and risk of bias. ${ }^{15}$ GRADE evidence profiles/summary of findings tables were generated using GRADEPro.

9. As per the ESO standard operating procedure, each PICO question was addressed by writing up to three distinct paragraphs. First, a paragraph named 'Analysis of current evidence', in which the results of the dedicated RCTs were summarized and briefly discussed. Where no RCT was 
available, this paragraph described results of systematic reviews of non-randomized studies. At the end of the first paragraph, an evidence-based recommendation was provided, based on the GRADE methodology. The direction, strength and formulation of the recommendation were determined according to the GRADE evidence profiles and the ESO standard operating procedure. Second, an 'Additional information' paragraph could be added to provide more details on randomized trials mentioned in the first paragraph, to summarize results of observational studies, or to provide information on ongoing or future trials. Third, according to the revised ESO standard operating procedure, ${ }^{14}$ an 'Expert consensus statement' paragraph was added whenever the PICO group deemed that the available evidence was insufficient to provide evidence-based recommendations for situations in which practical guidance is needed for routine clinical practice. In that particular case, a pragmatic suggestion was provided. Importantly, the suggestions provided in this paragraph should not be mistaken as evidence-based recommendations.

10. The present document was subsequently reviewed several times by all MWG members, and iteratively modified until a consensus was reached. Finally, the document was reviewed and approved by external reviewers and members of the ESO and ESMINT Guideline Boards and Executive Committees.

\section{RESULTS}

\section{Patients admitted to a thrombectomy-capable center ('mothership' paradigm)}

PICO 1: For large vessel occlusion acute ischemic stroke ( $\leq 4.5$ hours of symptom onset) patients directly admitted to a thrombectomy-capable center and eligible for both treatments, does mechanical thrombectomy alone compared with intravenous thrombolysis plus mechanical thrombectomy lead to:

1. a non-inferior proportion of patients with good outcome (mRS $0-2$ ) at 90 days?

2. non-inferior or better results on other efficacy outcomes (whole range of the mRS; mRS 0-1; successful reperfusion)?

3. a reduction in the risk of adverse events (mortality at 90 days, sICH, any $\mathrm{ICH}$ )?

4. a reduction in key time metrics?

\section{Analysis of current evidence}

The literature search identified four published RCTs addressing this PICO question. We also included the results of two unpublished RCTs which were recently presented at international conferences (tables 1 and 2).

The first published trial was Direct Intraarterial Thrombectomy in Order to Revascularize Acute Ischemic Stroke Patients with Large Vessel Occlusion Efficiently in Chinese Tertiary Hospitals Multicenter Randomized Clinical Trial (DIRECT-MT), which compared direct MT alone versus MT preceded by IVT with alteplase $(0.9 \mathrm{mg} / \mathrm{kg})$ administered within 4.5 hours after stroke onset in patients with anterior circulation LVO. ${ }^{6}$ A total of 654 LVO (ICA, M1 and M2 occlusions) patients from 41 academic tertiary care centers in China were analyzed. The primary analysis was based on reduced disability ( $\geq 1$ point reduction across all $\mathrm{mRS}$ scores at 90 days) and non-inferiority was defined on the basis of a lower boundary of the $95 \% \mathrm{CI}$ of the corresponding adjusted cOR equal to or larger than 0.80 . This predefined criterion was met (adjusted cOR mRS $1.07,95 \%$ CI 0.81 to 1.40 , $\mathrm{p}=0.04$ for non-inferiority). However, this non-inferiority margin was very liberal and the wide $\mathrm{CI}$ included both important harms and important benefits for either strategy. Furthermore, the comparison was confounded by time to treatments; a long door-to-IVT time (median $59 \mathrm{~min}$ ) and a very short delay from start of IVT to groin puncture (median approximately $27 \mathrm{~min}$ ) were documented in the bridging therapy group. In addition, the Chinese healthcare system requires initial self-pay for alteplase, followed by reimbursement from insurance when available to the patient. ${ }^{21}$ This may have resulted in a delay in consenting the patient, thereby delaying the door-to-IVT time (median $59 \mathrm{~min}$ ). Moreover, in the bridging therapy group, 31 patients did not receive MT and an additional 30 patients did not receive any or the full-dose of alteplase. In addition, multiple protocol violations (e.g. missing baseline CT angiography, lack of proof of occlusion on baseline CT angiography, crossovers between treatment arms) were not addressed in the published per-protocol analysis of the trial. ${ }^{22}$ The proportion of patients with successful reperfusion after thrombectomy (expanded TICI (eTICI) $\geq 2 \mathrm{~b}$ ) was $79.4 \%$ versus $84.5 \%$ (OR $0.70,95 \% \mathrm{CI} 0.47$ to 1.06$)$ in the direct MT and the bridging therapy groups, respectively. sICH occurred in $4.3 \%$ and $6.1 \%$ of patients in the direct MT and bridging therapy groups, respectively (RR $0.70,95 \%$ CI 0.36 to 1.37).

In the Direct Endovascular Thrombectomy vs Combined IVT and Endovascular Thrombectomy for Patients with Acute Large Vessel Occlusion in the Anterior Circulation (DEVT) trial, patients with LVO (ICA and M1 occlusions) acute ischemic stroke eligible for IVT were enrolled in 33 tertiary stroke centers in China and randomized within 4.25 hours of symptom onset to MT alone or bridging therapy with alteplase $0.9 \mathrm{mg} /$ $\mathrm{kg}$. The primary endpoint was the proportion of patients achieving good outcome at 90 days (mRS 0-2). The chosen non-inferiority margin in DEVT was an absolute difference of $10 \%$. The trial was stopped after randomization of 234 of 970 planned patients because non-inferiority was demonstrated. At 90 days, $63(54.3 \%)$ patients in the direct MT group and 55 $(46.6 \%)$ in the bridging therapy group achieved a good outcome (difference $7.7 \%$, one-sided $97.5 \% \mathrm{CI}-5.1 \%$ to $\infty$, $\mathrm{p}$ for noninferiority $=0.003$ ). No significant between-group differences in 90-day mortality ( $17.2 \%$ vs $17.8 \%$; difference $-0.5 \%, 95 \% \mathrm{CI}$ $-10.3 \%$ to $9.2 \%)$ and sICH (6.1\% vs $6.8 \%$; difference $-0.8 \%$, $95 \% \mathrm{CI}-7.1 \%$ to $5.6 \%)$ were observed. The proportion of patients with successful reperfusion after MT (eTICI $\geq 2 \mathrm{~b}$ ) was $88.5 \%$ versus $87.2 \%$ (adjusted OR $1.14,95 \%$ CI 0.50 to 2.61 ) in the direct MT and the bridging therapy groups, respectively. DEVT shares similar limitations with DIRECT-MT, including a generous non-inferiority margin and the Chinese reimbursement protocols before receiving alteplase as part of routine or study care. Again, the door-to-IVT time was long (median $61 \mathrm{~min}$ ), although onset to groin puncture times were similar between groups (200 vs $210 \mathrm{~min}$ ).

In the Direct Mechanical Thrombectomy in Acute LVO Stroke (SKIP) trial, 204 patients with ICA or M1 occlusion acute ischemic stroke eligible for IVT were enrolled in $23 \mathrm{MT}$-capable stroke centers in Japan and randomized to MT alone or bridging therapy with alteplase at the dose of $0.6 \mathrm{mg} / \mathrm{kg}$ within 4.5 hours of onset. ${ }^{7}$ The primary endpoint was the proportion of patients achieving good outcome at 90 days (mRS $0-2$ ), with a non-inferiority margin OR of 0.74 (lower boundary of the $\mathrm{CI}$ ), assessed using a one-sided significance threshold of 0.025 (97.5\% CI). SKIP did not demonstrate non-inferiority of direct MT versus bridging therapy (mRS 0-2: $59.4 \%$ vs $57.3 \%$; OR 1.09 , one-sided $97.5 \% \mathrm{CI} 0.63$ to $\infty$, one-sided $\mathrm{p}$ value for noninferiority $=0.18)$. Mortality at 90 days $(7.9 \%$ vs $8.7 \%)$ and sICH according to the SITS-MOST definition (5.9\% vs $7.7 \%$ ) did not 


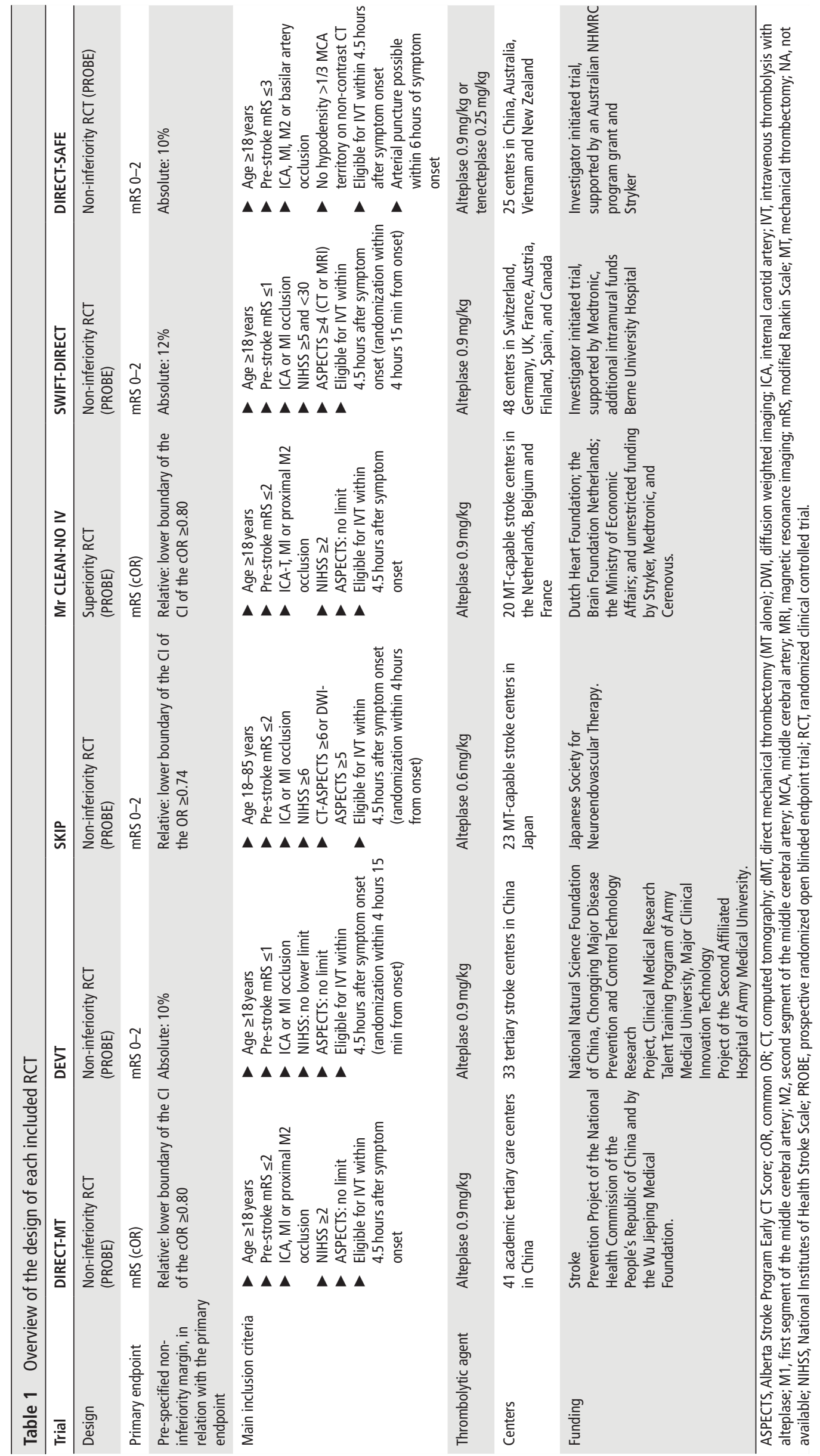




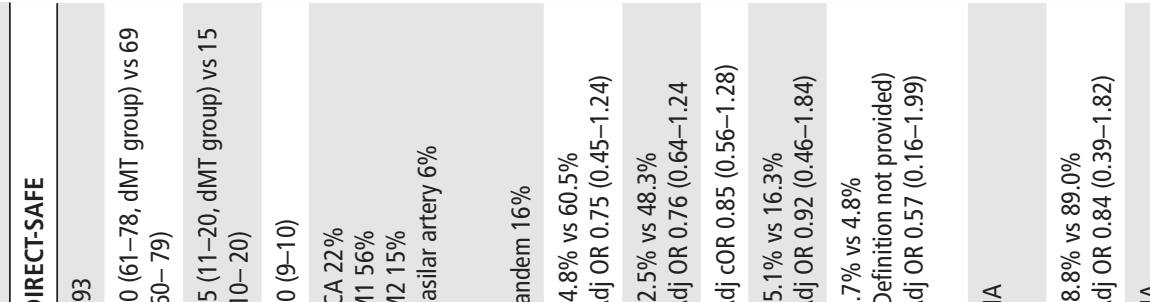

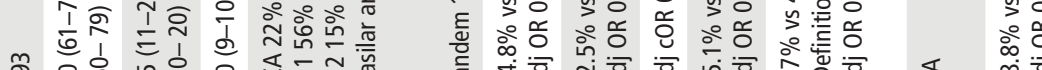
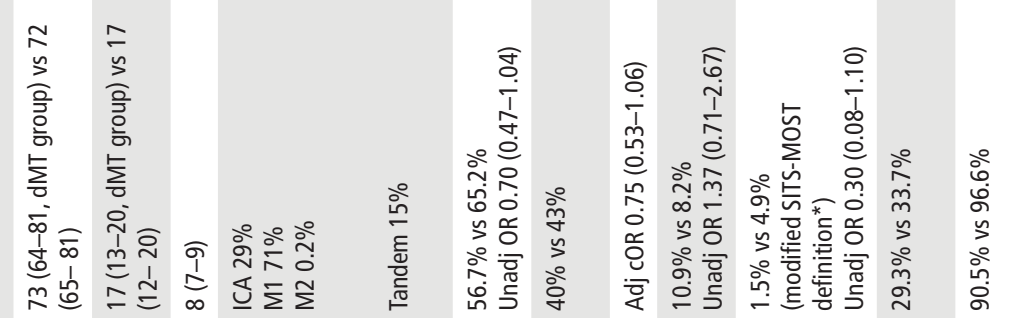

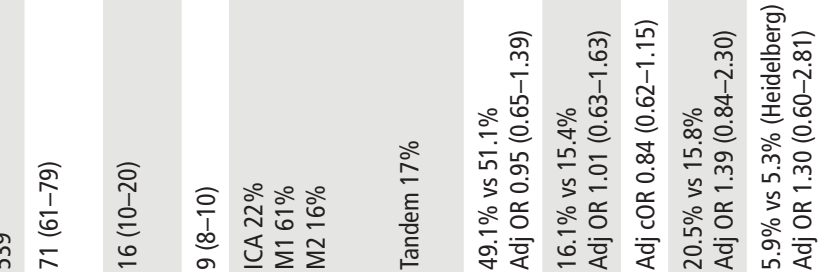

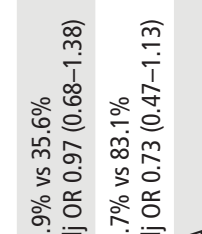

$\Sigma \Sigma$

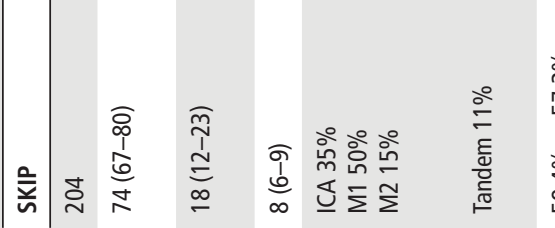

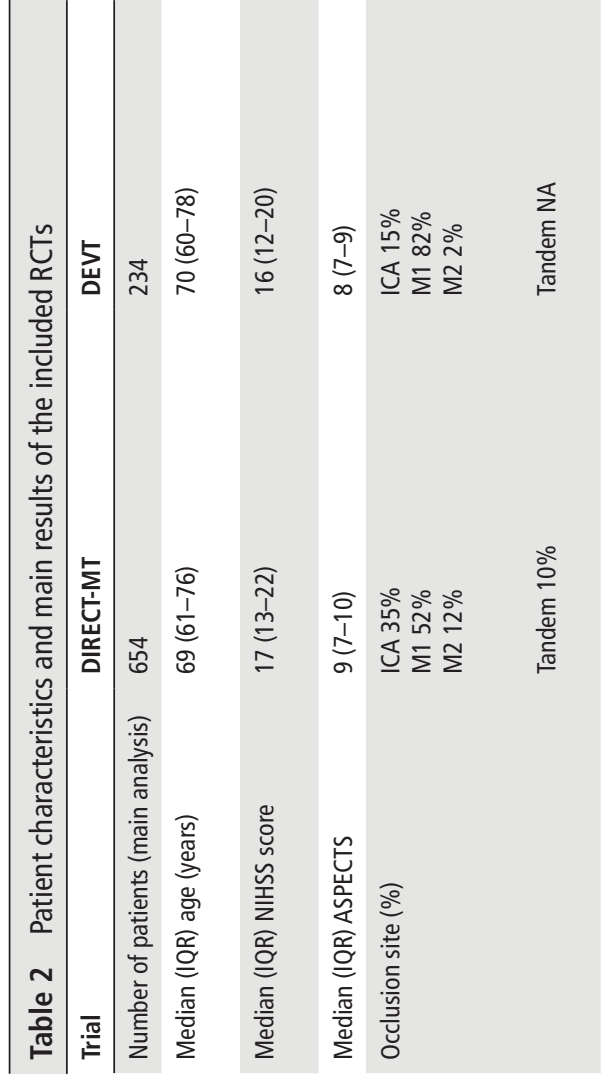

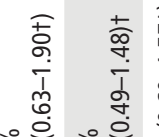

$\circ \circ \stackrel{m}{\circ}$ 崖余

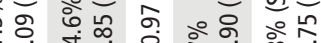

ñ

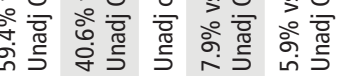

₹

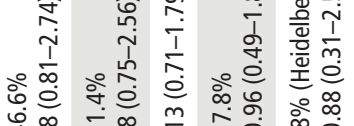

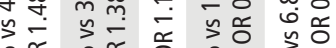

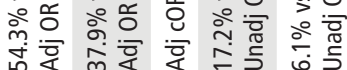

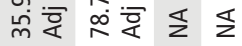

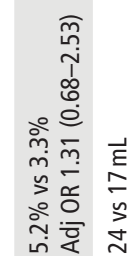

$\therefore \div$ 훙

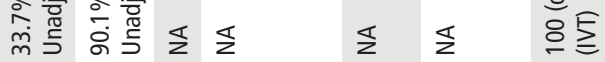

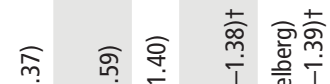

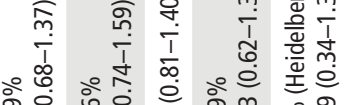

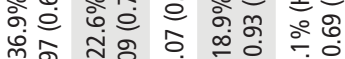
so ○. ว०

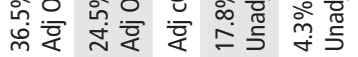

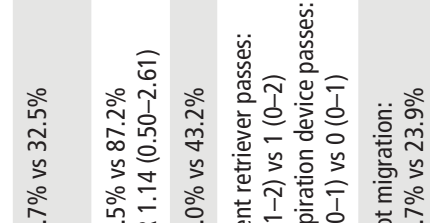

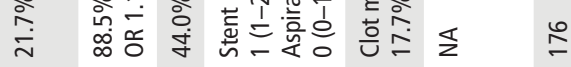
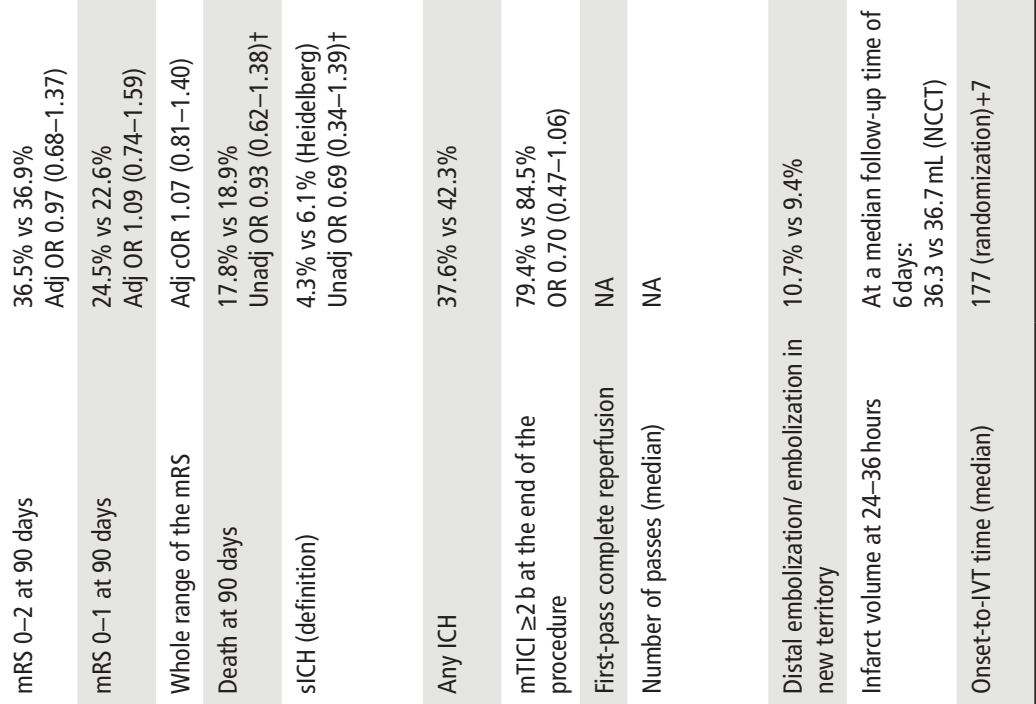
differ significantly between the two groups. The main limitations of this trial are the very liberal non-inferiority margin, the modest sample size, and the relatively long door-to-needle time probably delayed by patient consent and randomization. As a consequence, the time between IVT and arterial puncture was very short (median randomization-to-IVT and randomizationto-arterial puncture times of 14 and $22 \mathrm{~min}$, respectively). Of particular note, arterial puncture was performed before the start of IVT in $22(21.4 \%)$ patients in the bridging therapy group. The use of low-dose alteplase $(0.6 \mathrm{mg} / \mathrm{kg})$, which is not currently recommended in European and US guidelines, ${ }^{23} 24$ also limits the generalizability of the SKIP results.

Unlike previous RCTs, the primary aim of the Multicenter Randomized CLinical trial of Endovascular treatment for Acute ischemic stroke in the Netherlands- NO IV (MR CLEAN-NO IV) was to assess the superiority of direct MT over bridging therapy with alteplase $(0.9 \mathrm{mg} / \mathrm{kg})$ based on the adjusted $\mathrm{cOR}$ for reduced disability (i.e., $\geq 1$ point reduction across all $\mathrm{mRS}$ scores at 90 days). ${ }^{9}$ In the case that superiority could not be demonstrated, the lower boundary of the $95 \% \mathrm{CI}$ of this cOR was prespecified as equal to or larger than 0.80 to claim non-inferiority (ie, the same pre-specified margin as in the DIRECT-MT trial). A total of 539 patients with LVO (ICA, M1 and M2) acute ischemic stroke eligible for IVT within 4.5 hours of symptom onset were enrolled at 20 MT-capable stroke centers in the Netherlands, Belgium and France and included in the main analysis. MR CLEAN-NO IV failed to demonstrate both superiority and non-inferiority of direct MT over bridging therapy regarding functional outcome at 90 days (adjusted cOR $0.84,95 \%$ CI 0.62 to 1.15). Similar results were observed for secondary endpoints including dichotomizations of the mRS. Mortality (20.5\% vs $15.8 \%$; adjusted OR $1.39,95 \% \mathrm{CI} 0.84$ to 2.30 ) and sICH (5.9\% vs 5.3\%; adjusted OR $1.30,95 \%$ CI 0.60 to 2.81$)$ did not significantly differ between the direct MT and the bridging therapy groups. Finally, the rates of successful reperfusion on last angiographic run $=$ did not significantly differ between the two groups (78.7\% vs $83.1 \%$; adjusted OR $0.73,95 \% \mathrm{CI} 0.47$ to 1.13$)$.

The results of the Bridging Thrombolysis vs Direct Mechanical Thrombectomy in Acute Ischemic Stroke (SWIFT-DIRECT) trial were presented during the 2021 ESO Conference. ${ }^{11}$ SWIFTDIRECT sought to determine whether patients experiencing an acute ischemic stroke due to LVO in the anterior circulation (ICA and M1) would have a non-inferior functional outcome at 90 days when treated with direct MT compared with patients treated with bridging thrombolysis with alteplase at $0.9 \mathrm{mg} / \mathrm{kg}$ within 4.5 hours of onset. The primary endpoint was the proportion of patients achieving good outcome (mRS $0-2$ at 90 days). The non-inferiority margin was an absolute difference of $12 \%$. The use of a Solitaire device was mandatory, although additional devices were allowed. A total of 408 LVO patients from 48 stroke centers in Europe and North America were included (201 in the direct MT group and 207 in the bridging therapy group). SWIFT-DIRECT did not demonstrate non-inferiority. At 90 days, 111 (56.7\%) patients in the direct MT group and 135 (65.2\%) in the bridging therapy group achieved good outcome (adjusted risk difference $-7.3 \%$, lower limit of one-sided 95\% CI -15.1\%). The adjusted cOR for reduced disability with direct MT was 0.75 (95\% CI 0.53 to 1.06 ). The rates of mortality at 90 days were similar in patients treated with direct MT (11.0\%) and bridging therapy (8.5\%). Patients with direct MT tended to have lower rates of sICH compared with the bridging therapy group $(1.5 \%$ vs $4.9 \%, \mathrm{p}=0.09)$. Conversely, the rate of successful post-interventional reperfusion was higher in the bridging therapy group ( $96 \%$ vs $91 \%, \mathrm{p}=0.05)$. 


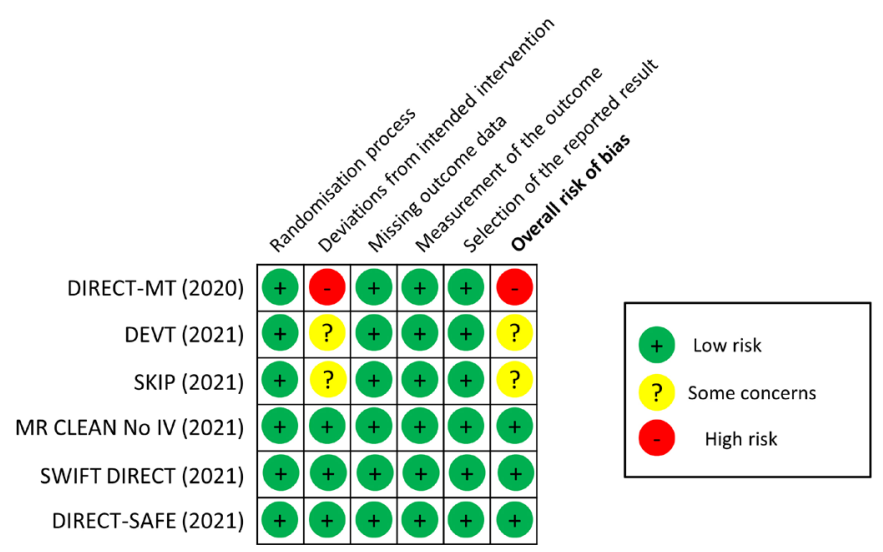

Figure 1 Risk of bias in each randomized controlled trial of MT alone versus IVT plus MT for anterior circulation large vessel occlusion ischemic stroke within 4.5 hours of symptom onset, with regards to mRS score at 90 days. IVT, intravenous thrombolysis with alteplase; mRS, modified Rankin Scale; MT, mechanical thrombectomy;

Finally, the results of DIRECT-SAFE (A Randomized Controlled Trial of DIRECT Endovascular Clot Retrieval vs Standard Bridging Thrombolysis With Endovascular Clot Retrieval) were presented at the 2021 World Stroke Congress. ${ }^{12}$ The aim of DIRECT-SAFE was to demonstrate the non-inferiority of direct MT over bridging therapy in patients with ICA, M1, M2 or basilar artery occlusion (the only one of the six RCTs to include any posterior circulation LVO). The primary trial outcome was good outcome defined as mRS 0-2 at 90 days or returning to premorbid mRS score, with a pre-specified absolute non-inferiority margin of $10 \%$. The authors also hypothesized that clinical outcome would differ between patients enrolled in Asian versus non-Asian regions. The use of a TREVO device was mandatory during the endovascular procedures. The study was terminated prematurely due to the publication of the results of DIRECT-MT, DEVT and SKIP. A total of 293 of 780 planned patients at 25 centers in Oceania and Asia were randomized and included in the intention-to-treat analysis. At 90 days, the primary outcome was achieved in $54.8 \%$ and $60.5 \%$ of patients in the direct MT and bridging therapy groups, respectively. Non-inferiority was not demonstrated (intention-to-treat risk difference $-5.1 \%$, $95 \% \mathrm{CI}-16.0 \%$ to $5.9 \%, \mathrm{p}=0.19$ ). Mortality rates at 90 days were similar in patients treated with direct MT and bridging therapy $(15.1 \%$ vs $16.3 \%, p=0.46)$. The rates of sICH did not differ significantly between the direct MT and the bridging therapy groups $(2.7 \%$ vs $4.8 \%, \mathrm{p}=0.38)$. The rates of successful post-interventional reperfusion were also similar at $89 \%$ (direct MT) versus $89 \%$ (bridging therapy) $(\mathrm{p}=0.66)$. In subgroup analyses, compared with bridging therapy, direct MT was associated with lower rates of good outcome in patients randomized in Asia compared with Oceania (adjusted OR 0.42, 95\% CI 0.21 to 0.86 vs $1.35,95 \% \mathrm{CI} 0.65$ to $2.80, \mathrm{p}$ for interaction $=0.02$ ).

MWG assessment of the risk of bias in each RCT according to the Cochrane RoB-2 tool with regards to $\mathrm{mRS}$ score at 90 days is presented in figure 1 . All studies were considered to be at overall low risk of bias except: (1) DIRECT-MT, which had a high risk of bias due to deviations from the intended intervention, as detailed above; (2) DEVT, due to concerns about a long door-to-IVT time, which are not in line with recommendations $^{25}$; and (3) SKIP, because more than $20 \%$ of patients in the bridging therapy group had arterial puncture before the start of IVT.

We conducted a study-level random-effects meta-analysis of the six RCTs of MT alone versus IVT plus MT, comprising 2331 patients. Compared with patients randomized to bridging therapy, the pooled unadjusted OR for good outcome in patients randomized to MT alone was 0.93 (95\% CI 0.79 to $1.10, p=0.38 ; I^{2}=0 \%$ ) (figure 2 ). The corresponding pooled $R R$ and risk difference were 0.96 (95\% CI 0.89 to $1.04, p=0.30$; $\left.\mathrm{I}^{2}=0 \%\right)$ and $-1.9 \%(95 \% \mathrm{CI}-5.9 \%$ to $2.1 \%)$ (figure 3$)$, respectively. Therefore, non-inferiority was not met based on our prespecified $1.3 \%$ margin. Importantly though, non-inferiority was also not met based on the maximum clinically acceptable non-inferiority margin of $5.0 \%$ proposed by MWG members. Similar results were obtained when risk difference was estimated with the random-effects pooled proportion of good outcome in the bridging therapy group $(52.8 \%)$ and the pooled $\mathrm{RR}$

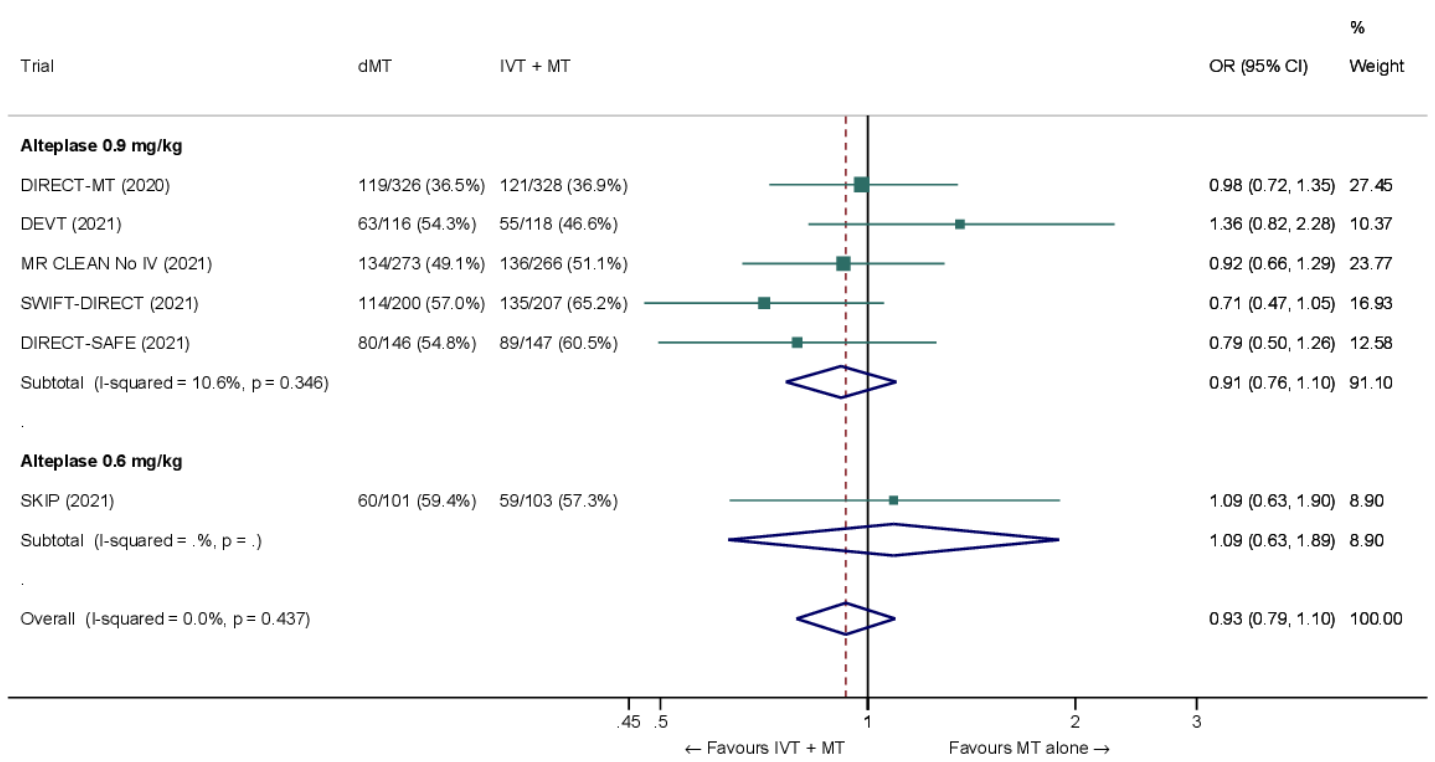

Figure 2 Good outcome (mRS 0-2 at 90 days) in 'mothership' anterior circulation large vessel occlusion stroke patients treated with MT alone versus IVT plus MT within 4.5 hours of symptom onset (unadjusted pooled OR, random-effects meta-analysis). dMT, direct mechanical thrombectomy (MT alone); IVT, intravenous thrombolysis with alteplase; mRS, modified Rankin Scale; MT, mechanical thrombectomy. 


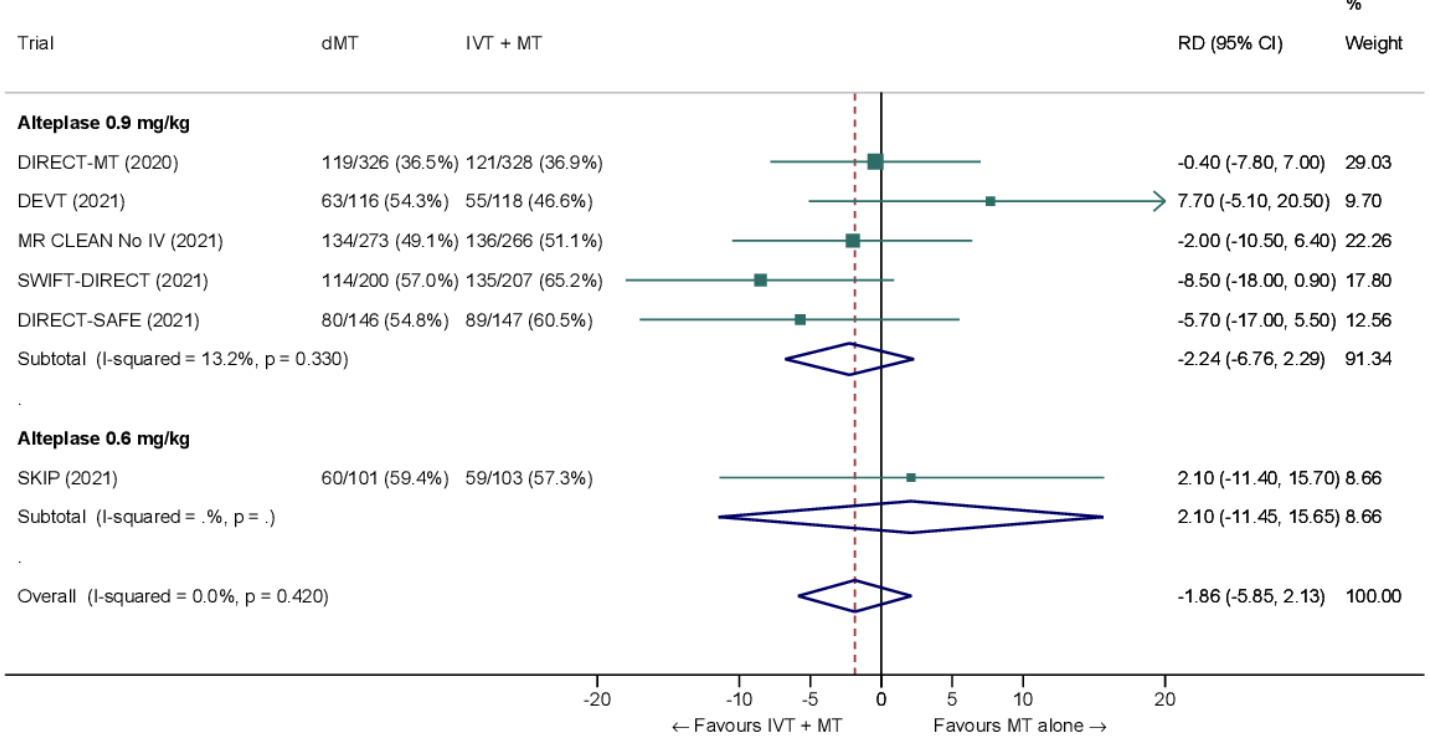

Figure 3 Pooled RD (in percent) for good outcome (mRS 0-2 at 90 days) in 'mothership' anterior circulation large vessel occlusion stroke patients treated with MT alone versus IVT plus MT within 4.5 hours of symptom onset (unadjusted pooled RD, random-effects meta-analysis). dMT, direct mechanical thrombectomy (MT alone); IVT, intravenous thrombolysis with alteplase; mRS, modified Rankin Scale; MT, mechanical thrombectomy; RD, risk difference.

for good outcome (risk difference $-2.1 \%$, 95\% CI $-6.3 \%$ to $2.1 \%$ ). Finally, in a sensitivity analysis based on a fixed effect model, the pooled risk difference was $-1.9 \%(95 \%$ CI $-5.9 \%$ to $2.1 \%$ ). The results did not significantly differ between studies conducted in Asia and in Europe/North America ( $\mathrm{p}$ for heterogeneity $=0.13$ ) (figure 4). A more detailed analysis on the role of ethnicity and national healthcare systems on the effects of IVT would require individual patient-level data.

The common adjusted OR for reduced disability with MT alone was 0.92 (95\% CI 0.80 to $1.07, \mathrm{p}=0.27 ; \mathrm{I}^{2}=0 \%$ ) (figure 5). The pooled unadjusted OR for excellent outcome
(mRS $0-1$ at 90 days) was 0.99 (95\% CI 0.82 to $1.18, \mathrm{p}=0.88$; $\left.\mathrm{I}^{2}=0 \%\right)$. The rates of all-cause mortality at 90 days were similar in patients randomized to MT alone and bridging therapy (unadjusted pooled OR $1.06,95 \%$ CI 0.84 to $1.35, p=0.60 ; \mathrm{I}^{2}=0 \%$ ) (figure 6). Successful reperfusion at the end of the endovascular procedure was significantly less frequent in patients randomized to MT alone (unadjusted pooled OR $0.72,95 \%$ CI 0.57 to 0.92 , $\mathrm{p}=0.01 ; \mathrm{I}^{2}=0 \%$ ) (figure 7 ). sICH did not differ significantly between treatment arms (unadjusted pooled OR $0.77,95 \% \mathrm{CI}$ 0.52 to $1.13, p=0.18 ; \mathrm{I}^{2}=0 \%$ ) (figure 8 ), but the occurrence of any $\mathrm{ICH}$ was less frequent in patients randomized to MT alone

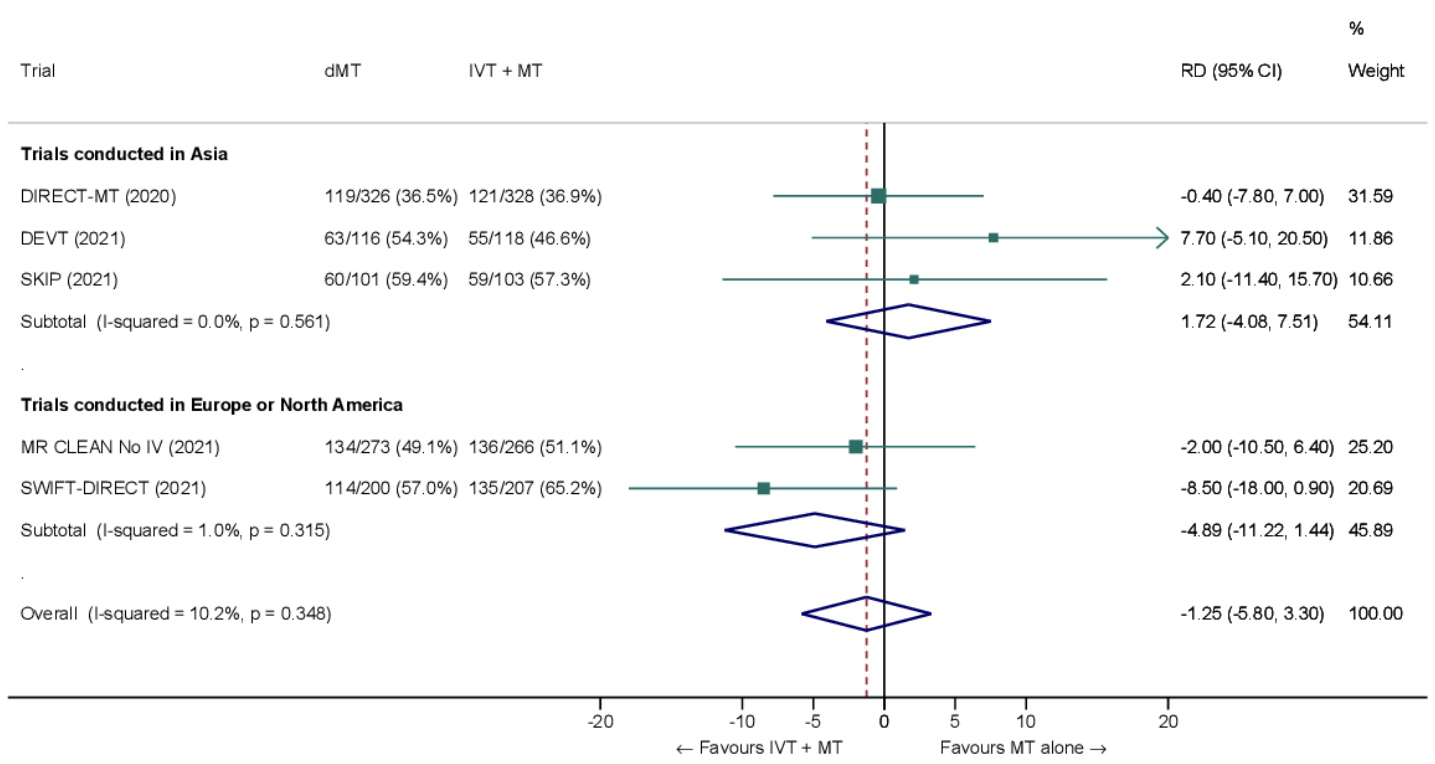

Figure 4 Pooled RD (in percent) for good outcome (MRS 0-2 at 90 days) in 'mothership' anterior circulation large vessel occlusion stroke patients treated with MT alone versus IVT plus MT within 4.5 hours of symptom onset, stratified by geographic region ( $p$ for heterogeneity between subgroups=0.13; unadjusted pooled RD, random-effects meta-analysis). DIRECT-SAFE ${ }^{12}$ was not included because it was conducted in Oceania (157 patients) and Asia (136 patients) and data allowing estimation of RD on functional outcome across geographical subgroups was not available at the time of the preparation of this expedited recommendation. dMT, direct mechanical thrombectomy (MT alone); IVT, intravenous thrombolysis with alteplase; mRS, modified Rankin Scale; MT, mechanical thrombectomy; RD, risk difference. 


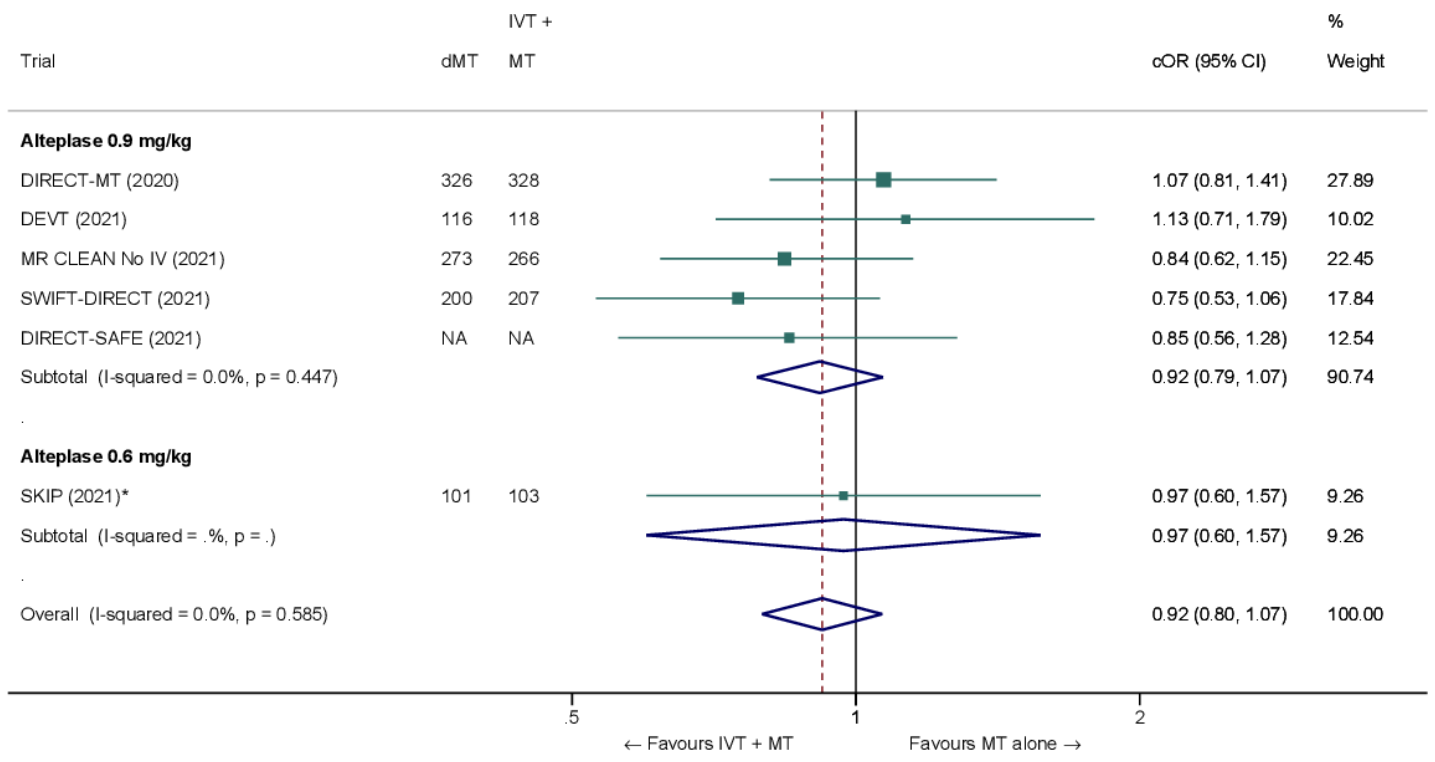

Figure 5 Pooled COR for reduced disability (improvement of a least 1 point on the mRS at 90 days) in 'mothership' anterior circulation large vessel occlusion stroke patients treated with MT alone versus IVT plus MT within 4.5 hours of symptom onset (adjusted pooled cOR, random-effects meta-analysis). *All cORs are adjusted except in the SKIP trial. Adjustment variables varied across studies. COR, common OR; dMT, direct mechanical thrombectomy (MT alone); IVT, intravenous thrombolysis with alteplase; mRS, modified Rankin Scale; MT, mechanical thrombectomy.

(unadjusted pooled OR $0.80,95 \%$ CI 0.66 to $0.96, p=0.02$; $\mathrm{I}^{2}=10 \%$ ) (figure 9). Insufficient data were available to conduct analyses for the other predefined outcomes, including time metrics (table 2).

Table 3 provides details regarding the assessment of the quality of evidence, which was judged to be moderate due to inconsistency, as two trials met their predefined criteria for non-inferiority, ${ }^{68}$ whereas the four remaining trials did not. ${ }^{7911} 12$

\section{Additional information}

Two large meta-analyses of observational studies evaluating the safety and efficacy of bridging therapy compared with direct MT have suggested that bridging therapy is associated with higher rates of post-intervention successful recanalization, higher rates of 90-day good outcome, and lower rates of 90 -day mortality without increased risk of $\mathrm{sICH} .{ }^{17}{ }^{26}$ Nevertheless, the results of these observational studies should be interpreted with caution because they are prone to selection bias (i.e. the majority of patients treated with direct MT had contraindications to alteplase) and residual confounding. Other meta-analyses including both RCTs and observational studies have also been published. ${ }^{27-30}$

For patients with LVO admitted within 6 hours after symptom onset, a recent RCT found that, compared with conventional workflow, the direct transfer to the angiography suite, and administration of alteplase in the suite if indicated, increased the odds of patients undergoing MT, decreased hospital workflow time, and improved clinical outcome. ${ }^{31}$

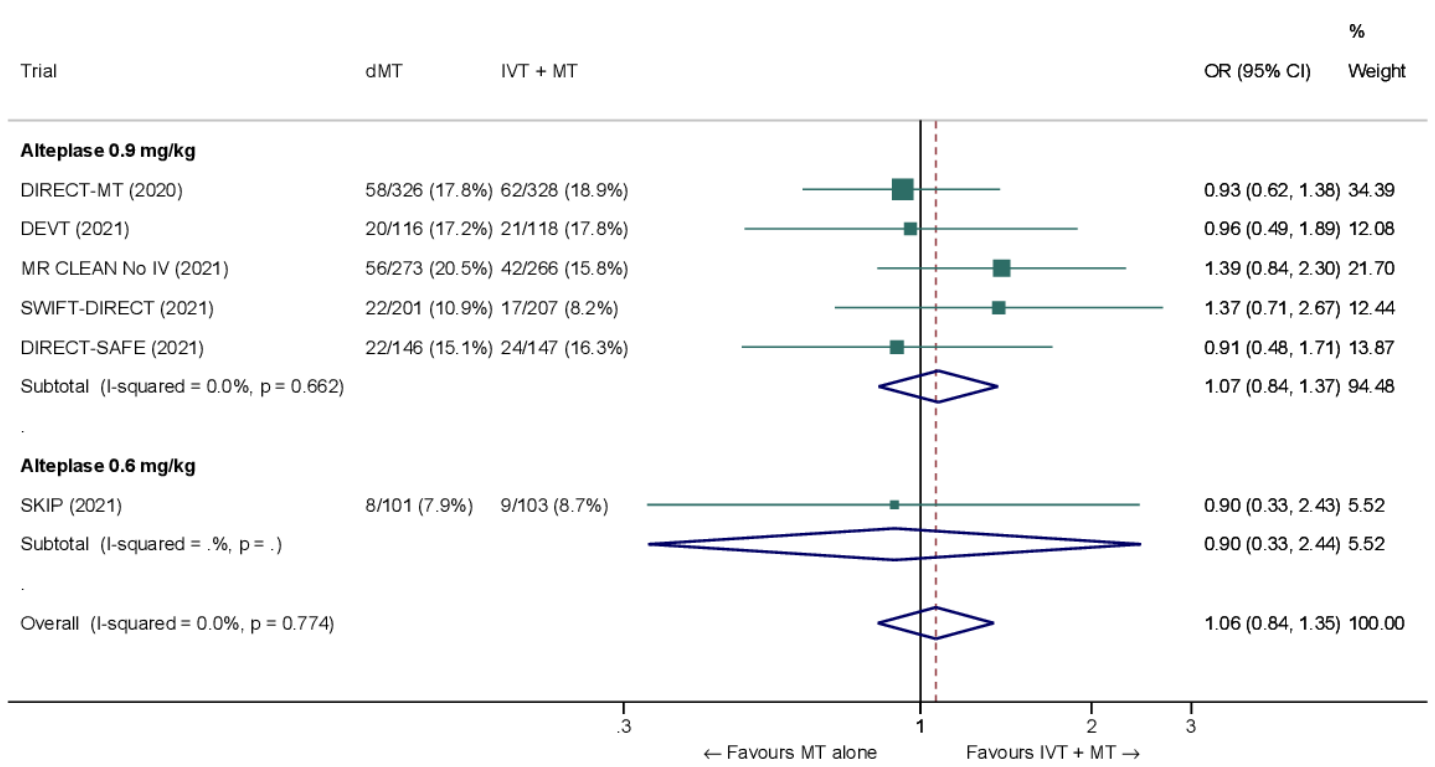

Figure 6 All-cause mortality at 90 days in 'mothership' anterior circulation large vessel occlusion stroke patients treated with MT alone versus IVT plus MT within 4.5 hours of symptom onset (unadjusted pooled OR, random-effects meta-analysis). dMT, direct mechanical thrombectomy (MT alone); IVT, intravenous thrombolysis with alteplase; MT, mechanical thrombectomy. 


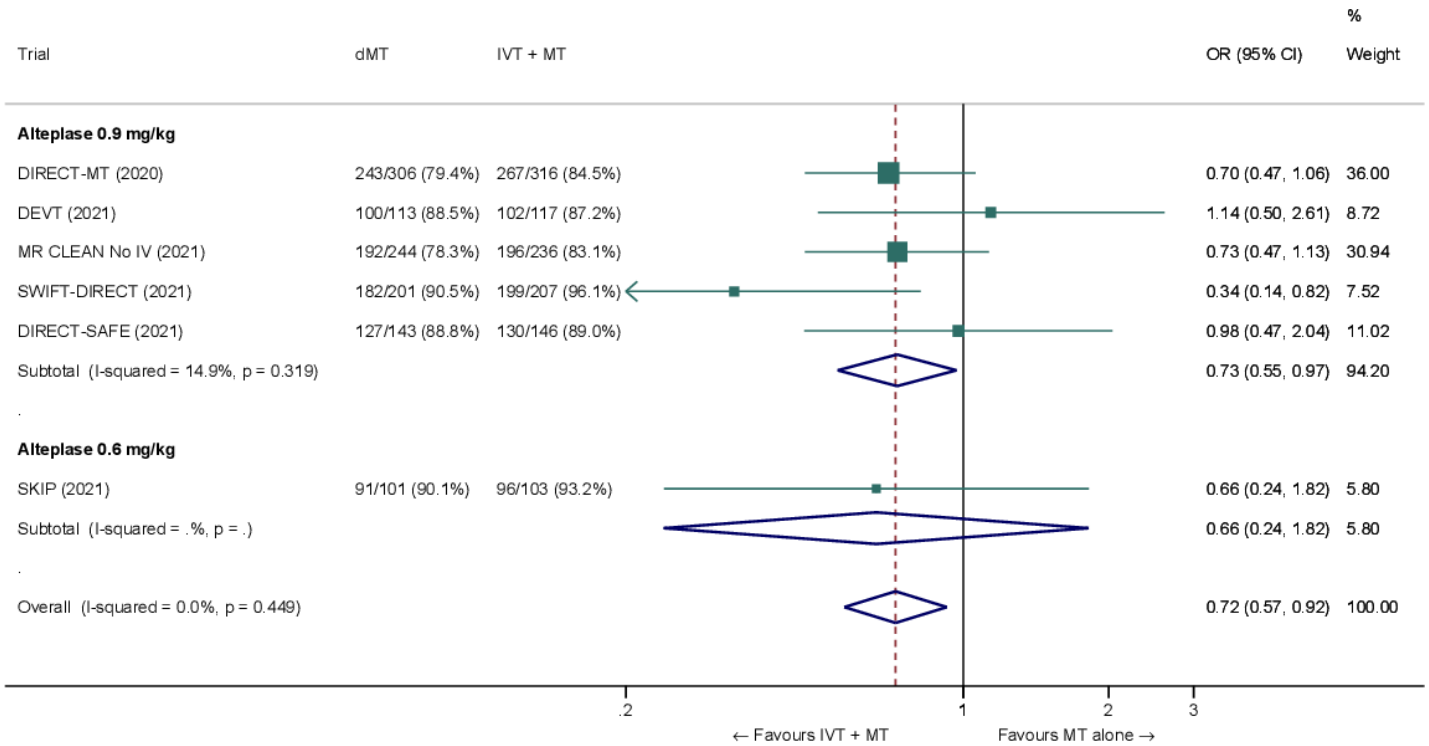

Figure 7 Pooled OR for successful reperfusion $(\mathrm{mTICl} \geq 2 \mathrm{~b})$ at the end the endovascular procedure in 'mothership' anterior circulation large vessel occlusion stroke patients treated with MT alone versus IVT plus MT within 4.5 hours of symptom onset (unadjusted pooled OR, random-effects metaanalysis). dMT, direct mechanical thrombectomy (MT alone); IVT, intravenous thrombolysis with alteplase; MT, mechanical thrombectomy; mTICl, modified Treatment In Cerebral Ischemia.

\section{Expert consensus statement}

The six recently published and presented RCTs studying the effect of IVT with alteplase before MT all included patients eligible for both treatments, and required IVT to be administered within 4.5 hours of stroke onset. ${ }^{6-83233}$ The results of these trials are therefore valid for patients who can be treated within this time window.

However, in 2018 and 2019, after the above mentioned trials were started, two randomized trials showed that a selected group of patients identified by advanced imaging who arrive more than 4.5 hours after symptom onset or last known well time may benefit from IVT. ${ }^{34}$
WAKE-UP was a placebo-controlled, randomized trial of patients aged 18 to 80 years who were more than 4.5 hours since last known well, had an unknown time of stroke onset, and could be treated within 4.5 hours of symptom recognition (ie, waking up or first seen with symptoms), of whom the majority (89\%) woke up with stroke symptoms. ${ }^{3}$ Eligible patients had to have diffusion weighted imaging-fluid attenuated inversion recovery (DWI-FLAIR) mismatch on MRI, a NIHSS score $\leq 25$, and a DWI lesion smaller than one third of the territory of the middle cerebral artery. Approximately 20\% of enrolled patients had an LVO. Patients were excluded if MT was planned, although MT at 6 or more hours from onset was not proven beneficial until

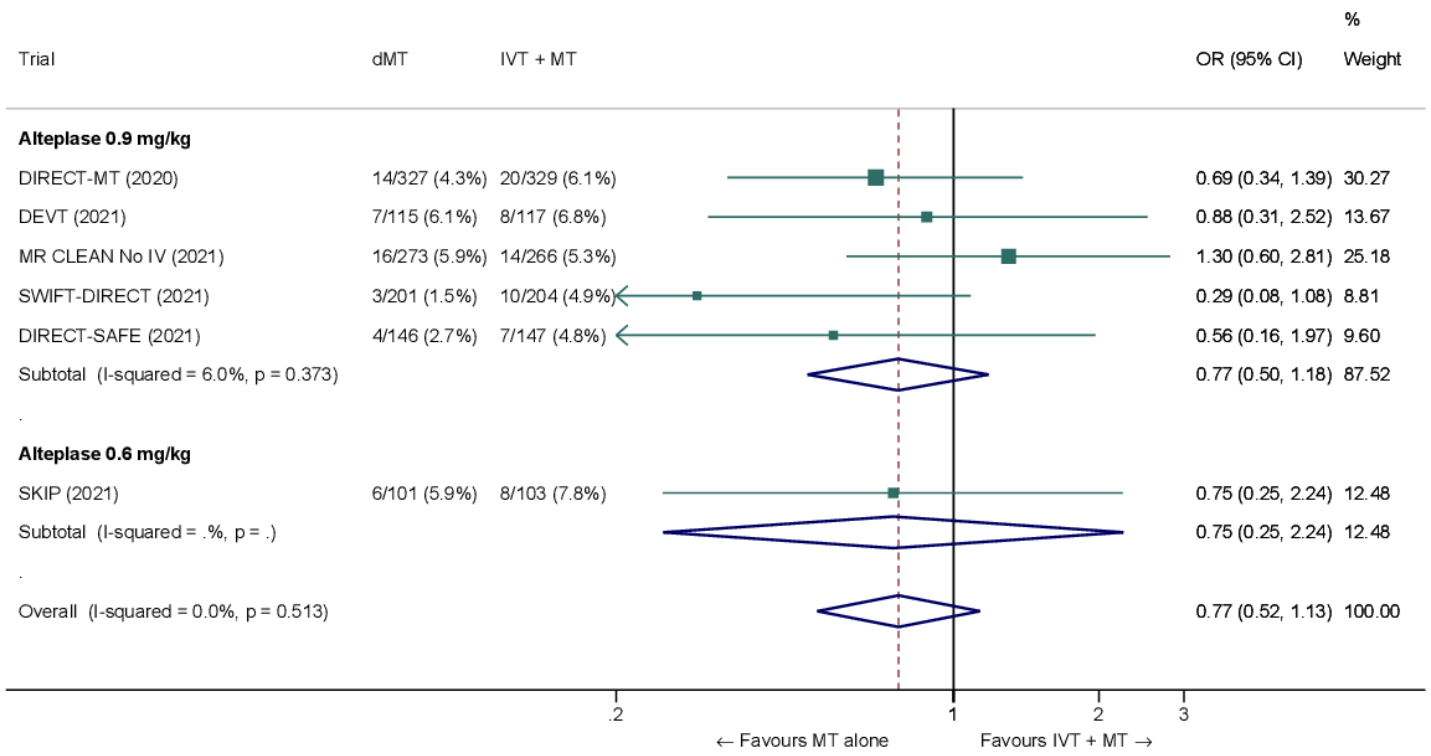

Figure 8 Pooled OR for sICH in 'mothership' anterior circulation large vessel occlusion stroke patients treated with MT alone versus IVT plus MT within 4.5 hours of symptom onset (unadjusted pooled OR, random-effects meta-analysis). The definition of sICH varied across studies (see table 2 for details). dMT, direct mechanical thrombectomy (MT alone); IVT, intravenous thrombolysis with alteplase; MT, mechanical thrombectomy; sICH, symptomatic intracerebral hemorrhage. 


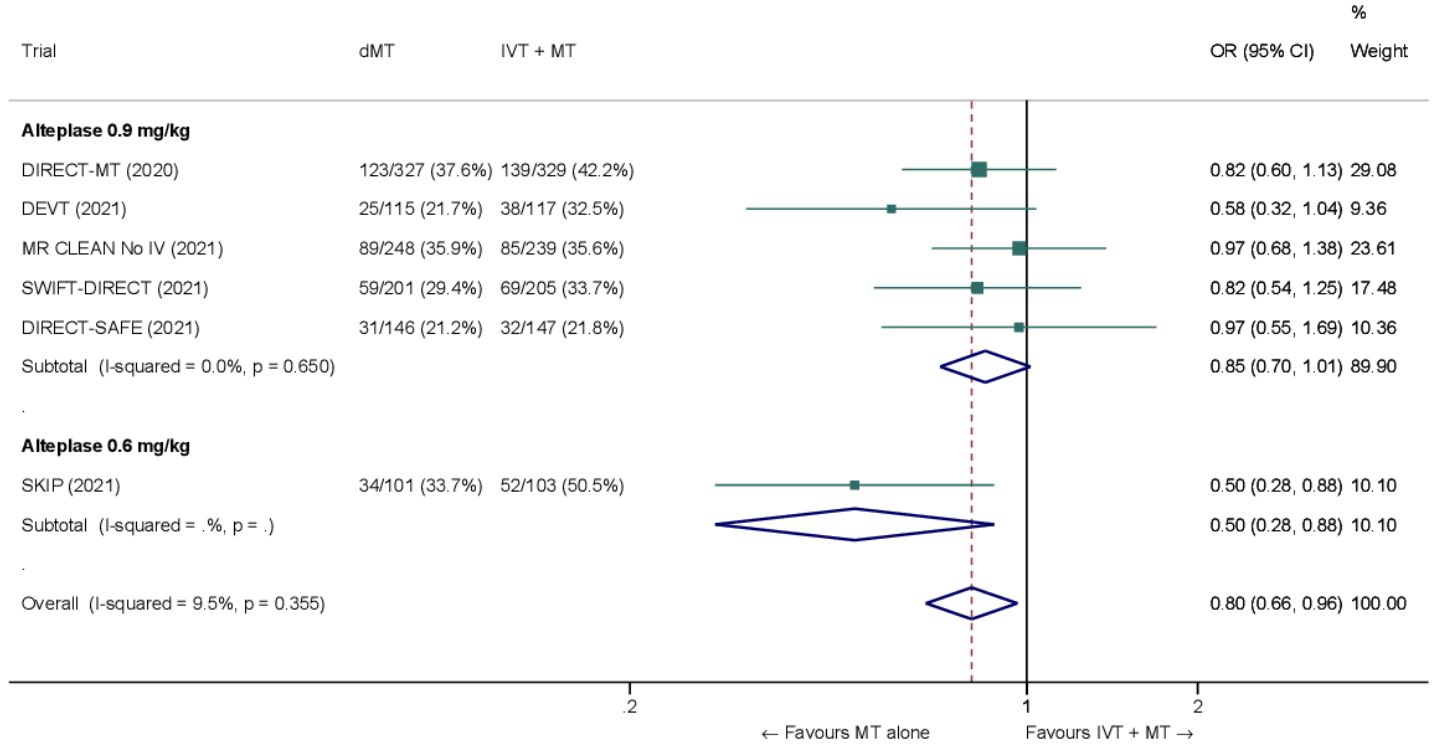

Figure 9 Pooled OR for any intracranial hemorrhage in 'mothership' anterior circulation large vessel occlusion stroke patients treated with MT alone versus IVT plus MT within 4.5 hours of symptom onset (unadjusted pooled OR, random-effects meta-analysis). dMT, direct mechanical thrombectomy (MT alone); IVT, intravenous thrombolysis with alteplase; MT, mechanical thrombectomy.

after the trial was completed. Compared with placebo, IVT was associated with a higher rate of excellent outcome (mRS $0-1$, $53 \%$ vs $42 \%$; adjusted OR $1.61,95 \%$ CI 1.09 to $2.36, \mathrm{p}=0.02$ ). IVT was also associated with a non-significantly increased risk of sICH $(2.0 \%$ vs $0.4 \%, p=0.15)$ and a non-significantly higher mortality at 90 days $(4.1 \%$ vs $1.2 \%, \mathrm{p}=0.07)$.

EXTEND was a randomized placebo-controlled trial involving patients with acute ischemic stroke (NIHSS score 4 to 26), in whom the assigned intervention was initiated between 4.5 and 9.0 hours after the onset of stroke $35 \%$ of the 225 included patients) or on awakening with stroke symptoms (if within 9 hours from the midpoint of sleep). ${ }^{4}$ Eligible patients had to have perfusion core/penumbra mismatch on CT or MRI. ${ }^{4}$ Patients were ineligible if MT was planned and again this trial commenced before MT was proven beneficial in the extended time window. Seventy-one percent of enrolled patients had an LVO. Compared with placebo, IVT was associated with a higher proportion of patients with excellent outcome (mRS $0-1$; adjusted RR $1.44,95 \% \mathrm{CI} 1.01$ to $2.06, \mathrm{p}=0.04$ ), and there was no evidence of treatment effect modification by different time intervals (4.5-6hours or 6-9 hours), or in patients with wake-up stroke ( $\mathrm{p}$ for interaction=0.41). A secondary prespecified ordinal analysis did not show a significant difference in functional outcome (common OR for reduced disability 1.55, $95 \%$ CI 0.96 to 2.49 ). IVT was associated with non-significantly higher rates of 90-day mortality (adjusted RR 1.17, 95\% CI 0.57 to 2.40) and sICH (adjusted RR 7.22, 95\% CI 0.97 to 53.54).

In a subsequent individual patient data meta-analysis including data from the perfusion imaging-based ECASS-4 and EPITHET trials ( $n=414$; wake-up strokes $51 \%$; LVO 61\%), ${ }^{3435}$ IVT was associated with higher rates of excellent outcome (OR $1.86,95 \% \mathrm{CI} 1.15$ to $2.99, \mathrm{p}=0.01)$, higher rates of $\mathrm{sICH}(5 \%$ vs $<1 \%$; OR 9.7, 95\% CI 1.23 to $76.55, p=0.03$ ) and no significant difference in mortality (OR $1.55,95 \%$ CI 0.81 to 2.96 , $\mathrm{p}=0.19) .{ }^{36}$ There was no evidence of a modification of the effect of IVT across three predefined time strata (4.5-6hours, 6-9 hours, wake-up stroke; $\mathrm{p}$ for interaction $=0.87$ ) or in patients with or without LVO ( $\mathrm{p}$ for interaction $=0.66$ ).
Another individual patient data meta-analysis included 843 patients with unknown stroke onset (on awakening in $89 \%$ of cases) enrolled in RCTs based on DWI-FLAIR mismatch (WAKE$\mathrm{UP}^{3}$ and THAWS ${ }^{37}$ ) or core/penumbra mismatch on perfusion MRI or CT (EXTEND ${ }^{4}$ and ECASS-4). ${ }^{34}{ }^{38}$ Compared with placebo or standard care, IVT was significantly associated with excellent outcome (adjusted OR 1.49, 95\% CI 1.10 to 2.03, $\mathrm{p}=0.01$ ) and reduced disability at 90 days (adjusted cOR 1.39 , $95 \% \mathrm{CI} 1.05$ to $1.80, \mathrm{p}=0.02$ ), at the expense of a higher risk of sICH ( $3 \%$ vs $0.5 \%$; adjusted OR $5.58,95 \%$ CI 1.22 to 25.50 , $\mathrm{p}=0.02$ ) and mortality within 3 months (adjusted OR 2.06, $95 \%$ CI 1.03 to $4.09, \mathrm{p}=0.04$ ). The effect of IVT was consistent across predefined subgroups, including imaging modality (CT vs MRI, $\mathrm{p}$ for interaction $=0.28$ ), wake-up stroke versus other situations ( $\mathrm{p}$ for interaction $=0.76$ ), and LVO status ( $\mathrm{p}$ for interaction $=0.28$ ). Of note, MT was not performed in the $25 \%$ of included patients with LVO.

Based on the results of the WAKE-UP, ${ }^{3}$ THAWS, ${ }^{37}$ EXTEND, ${ }^{4}$ ECASS- $4,{ }^{34}$ and EPITHET ${ }^{35}$ trials and their meta-analyses, ${ }^{36}{ }^{38}$ the recently published ESO guidelines on intravenous thrombolysis for acute ischemic stroke included recommendations for IVT beyond 4.5 hours after stroke onset or for patients with wake-up stroke or stroke of unknown onset time ${ }^{23}$ : IVT is recommended for patients with known stroke duration of 4.5 to 9 hours and for patients with stroke symptoms on awakening from sleep and their midpoint of sleep no longer than 9 hours before imaging with CT- or MRI- core/perfusion mismatch. IVT is also recommended for wake-up stroke patients who were last seen well more than 4.5 hours earlier with DWI-FLAIR mismatch on MRI. These ESO recommendations are for patients for whom MT is either not indicated or not planned.

The MWG members have provided below an expert consensus statement for IVT before MT in patients who wake up with stroke symptoms. As the WAKE-UP and THAWS trials included patients $<4.5$ hours after symptom recognition or awakening, and EXTEND and ECASS 4 included patients $<9$ hours after 'midtime of sleep' in case of ischemic stroke at awakening (which in most cases will correspond to $<4.5$ hours after awakening), the 


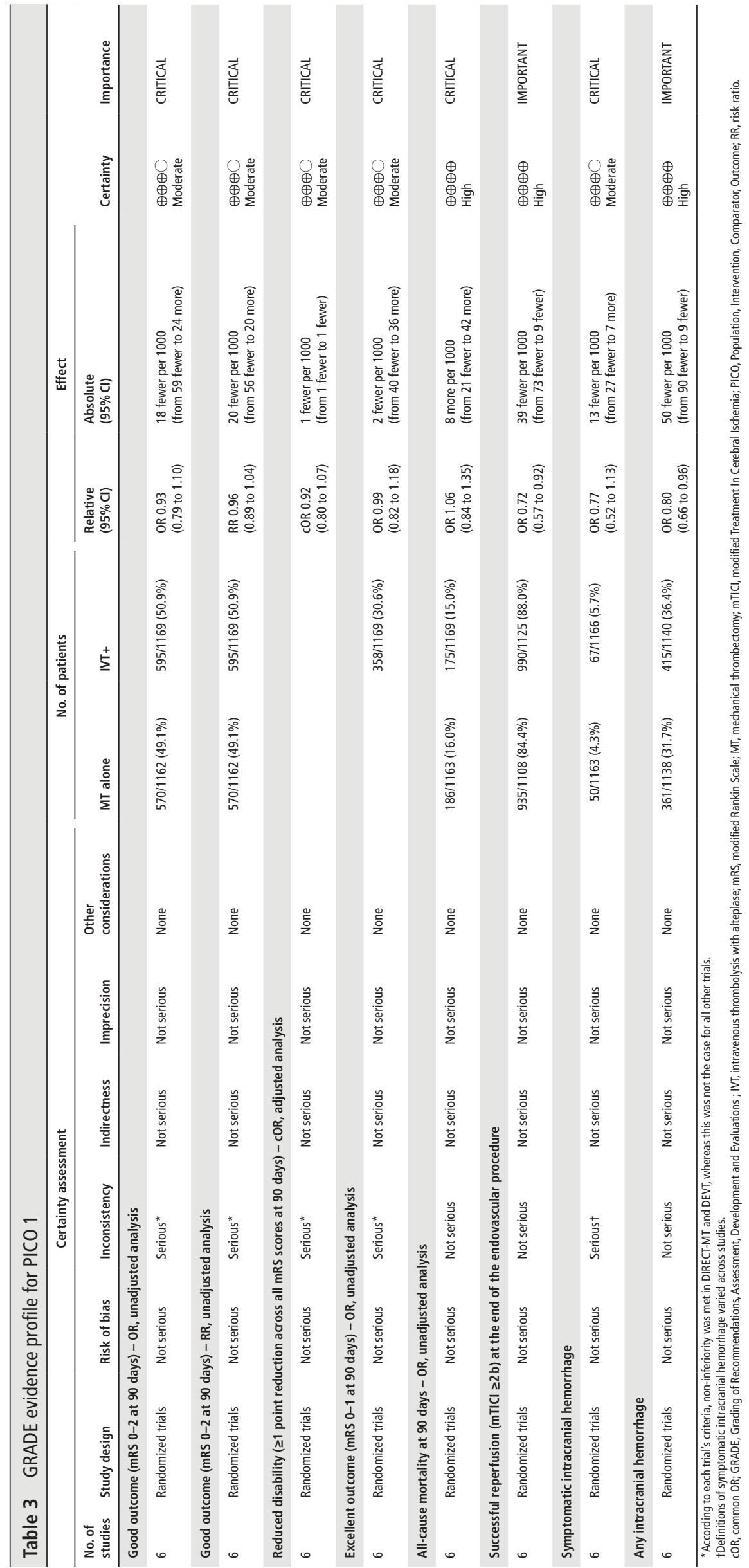


Evidence-based recommendation

For patients directly admitted to a thrombectomy-capable center for an acute ischemic stroke ( $\leq 4.5$ hours of symptom onset) with anterior circulation large vessel occlusion and who are eligible for both treatments, we recommend intravenous thrombolysis plus mechanical thrombectomy over mechanical thrombectomy alone.

Both treatments should be performed as early as possible after hospital arrival. Mechanical thrombectomy should not prevent the initiation of intravenous thrombolysis, and intravenous thrombolysis should not delay mechanical thrombectomy.

Quality of evidence: Moderate $\oplus \oplus \oplus$

Strength of recommendation: Strong $\uparrow \uparrow$

MWG members limit this expert consensus statement to patients arriving $<4.5$ hours after awakening. The results of the votes for this statement are provided in online supplemental table 2 . This expert consensus statement supersedes the one provided in the 2021 ESO guideline on intravenous thrombolysis for patients with wake-up stroke who are eligible for both IVT and MT. ${ }^{23}$

The ongoing Tenecteplase in Wake-up Ischaemic Stroke Trial (TWIST; NCT03181360) includes patients who present with a wake-up stroke within 4.5 hours after awakening, without any advanced imaging selection. ${ }^{39}$ The randomized Tenecteplase in Stroke Patients Between 4.5 and 24 Hours (TIMELESS; NCT03785678) will provide data on the comparison of tenecteplase and placebo in patients with LVO and penumbral tissue. Results from these two trials may shed new light on the effect of IVT with tenecteplase before MT.

Patients admitted to a center without thrombectomy facilities ('drip and ship' paradigm)

PICO 2: For large vessel occlusion acute ischemic stroke ( $\leq 4.5$ hours of symptom onset) patients admitted to a nonthrombectomy-capable center and eligible for both treatments, does mechanical thrombectomy alone compared with intravenous thrombolysis plus mechanical thrombectomy lead to:

\section{Expert consensus statement}

For patients directly admitted to a thrombectomy-capable center within 4.5 hours of symptom recognition after wake-up ischemic stroke caused by anterior circulation large vessel occlusion, we suggest intravenous thrombolysis plus mechanical thrombectomy over mechanical thrombectomy alone in selected patients.

The selection criteria for IVT and MT for patients with wake-up stroke are detailed in the corresponding European guidelines. ${ }^{53}$ Notably, eligibility imaging criteria for IVT include DWI-FLAIR mismatch or perfusion core/penumbra mismatch*.

*Perfusion core/penumbra mismatch:

- Infarct core ${ }^{* *}$ volume $<70 \mathrm{~mL}$

- and critically hypoperfused ${ }^{\dagger}$ volume/infarct core volume $>1.2$

- and mismatch volume $>10 \mathrm{~mL}$

* * Relative cerebral blood flow (rCBF) $<30 \%$ (CT perfusion) or apparent diffusion coefficient (ADC) $<620 \mu \mathrm{m}^{2} / \mathrm{s}$ (diffusion MRI)

${ }^{\dagger}$ Tmax $>6 \mathrm{~s}$ (perfusion CT or perfusion MRI)
1. a non-inferior proportion of patients with good outcome (mRS 0-2) at 90 days?

2. non-inferior or better results on other efficacy outcomes (whole range of the mRS; mRS 0-1; successful reperfusion)?

3. a reduction in the risk of adverse events (mortality at 90 days, sICH, any ICH)?

4. a reduction in key time metrics?

\section{Analysis of current evidence}

The literature search did not identify any RCT comparing the efficacy and safety of MT alone and bridging therapy in patients admitted to stroke centers with the capability to administer IVT but not MT. There are important theoretical arguments in favor of IVT pre-treatment in the 'drip and ship' model. Swift IVT delivery is associated with faster and more frequent IVT-induced recanalization and consequently better functional outcomes in acute ischemic stroke patients with LVO, as shown in the prospective CLOTBUST-PRO study. ${ }^{40}$ Moreover, a metaregression analysis in a meta-analysis comparing the efficacy of 'drip and ship' versus 'mothership' models revealed a significant inverse association between onset-to-needle and 90-day good outcome, with longer onset-to-needle time being detrimental for functional recovery. ${ }^{41}$ In addition, direct access to MT is limited to a minority of LVO patients, ${ }^{42} 43$ and withholding IVT in patients presenting to primary stroke centers may result in 'denial' of any reperfusion therapy in some patients who will reach the comprehensive stroke centers outside the time window for endovascular therapies and in those with unsuccessful MT. ${ }^{4445}$ Finally, the proportion of LVO 'drip and ship' patients who may successfully recanalize during transfer to comprehensive centers without receiving MT is approximately nine-fold higher in bridging therapy compared with direct MT $(11.7 \%$ vs $1.3 \%$ ), according to the findings of a single-center German study. ${ }^{46}$ In the French multicenter PREDICT-RECANAL study of 686 IVT-treated patients referred for MT, early recanalization (mTICI $\geq 2$ b) after IVT was independently associated with the drip-and-ship paradigm (adjusted OR 3.3, 95\% CI 1.8 to 6.0 ), with respective recanalization rates of $24.2 \%$ and $11.1 \%$ in drip-and-ship and mothership patients after centralized reading of arterial images. ${ }^{47}$ Similarly, a Swiss study reported higher complete recanalization rates before MT in IVT-treated patients according to the drip-and-ship model compared with the mothership model (13.6\% vs 6.2). ${ }^{48}$

According to a recent systematic review, few observational studies provide a comparison of direct MT and bridging therapy in 'drip-and-ship' patients. ${ }^{17}$ A subgroup analysis of the SELECT (Optimizing Patient Selection for Endovascular Treatment in Acute Ischemic Stroke) study evaluated LVO patients who were 'dripped' in primary stroke centers and 'shipped' to MT-capable centers. This analysis found that the rates of excellent functional outcome ( $\mathrm{mRS}$ scores of $0-1$ ) were significantly higher in patients receiving bridging therapy (36\%) compared with direct MT $(10 \%) .{ }^{49}$ The investigators documented an overall shift toward better functional outcomes with bridging therapy compared with direct MT (adjusted cOR 4.51, 95\% CI 1.44 to 14.15). The rates of 90 -day good functional outcome were numerically but non-significantly higher in the bridging therapy group (47\% vs $29 \%, \mathrm{p}=0.14)$; similarly, the rates of 90 -day mortality were numerically but non-significantly lower in the bridging therapy group ( $13 \%$ vs $29 \%, p=0.10)$, while the two groups had similar sICH rates. ${ }^{49}$ These findings were reproduced by a single-center German study that reported higher rates of excellent functional outcome (mRS scores $0-1$ or return to prestroke mRS-score) in 'drip and ship' patients receiving bridging therapy (23\% vs $14 \%$ 
for direct MT). ${ }^{46}$ There were no differences in the two groups with regard to safety outcomes including mortality and sICH. Finally, an analysis of the French Endovascular Treatment in Ischemic Stroke (ETIS) registry ${ }^{50}$ reported that among 1507 patients with anterior circulation LVO stroke treated with MT at three comprehensive stroke centers, 975 (64.7\%) received prior IVT. In the 'drip-and-ship' subgroup (70\% of the whole cohort), good outcome was observed in $50.3 \%$ and $39.7 \%$ of patients treated with bridging therapy and direct MT, respectively (unadjusted OR $1.54,95 \%$ CI 1.14 to $2.07, p=0.005$ ). However, this association was no longer significant after propensity score matching (OR $1.25,95 \%$ CI 0.86 to $1.82, \mathrm{p}=0.25$ ).

We conducted a meta-analysis of observational studies comparing direct MT with bridging therapy in drip-and-ship patients (table 4). ${ }^{46} 4950$ The pooled ORs for the association between direct MT and good and excellent functional outcomes were 0.63 (95\% CI 0.48 to $0.83, \mathrm{p}=0.001$ ) (figure 10 ) and 0.42 ( $95 \%$ CI 0.16 to $1.09, \mathrm{p}=0.07 ; \mathrm{I}^{2}=45 \%$ ) (figure 11 ), respectively. The probability of reduced disability was lower in the direct MT group compared with the bridging therapy group (adjusted cOR $0.22,95 \%$ CI 0.07 to $0.69, \mathrm{p}=0.01$ ), but this result was based on a single study. ${ }^{49}$ Direct MT was not significantly associated with all-cause mortality at 90 days (OR 1.42, $95 \% \mathrm{CI} 0.59$ to $3.44, \mathrm{p}=0.43 ; \mathrm{I}^{2}=56 \%$ figure 12 ). In a sensitivity analysis in which propensity score matching results from the study by Di Maria et al were used instead of unadjusted results, ${ }^{50}$ the pooled OR for mRS $0-2$ was 0.75 (95\% CI 0.53 to $\left.1.07, \mathrm{p}=0.11 ; \mathrm{I}^{2}=0 \%\right)$. Table 4 provides details regarding the assessment of the quality of evidence, which was judged to be low. Insufficient data were available to conduct analyses for most of our predefined outcomes.

In view of the above literature considerations and in light of the neutral results of a first RCT investigating prehospital triage for patients with suspected LVO, reporting similar outcomes in 'drip and ship' and 'mothership' models, ${ }^{51}$ we recommend that all IVT-eligible anterior circulation LVO patients presenting to stroke centers without endovascular facilities should receive IVT before their transfer to MT-capable centers according to current international recommendations. ${ }^{23}{ }^{24}$ Shorter door-in to door-out times in the primary stroke centers are also recommended to shorten onset-to-groin-puncture time, another important determinant of functional outcome. ${ }^{52}$

\section{Additional information}

Prior IVT guidelines have deemed the quality of evidence to be high for alteplase compared with placebo within 4.5 hours of acute ischemic stroke, and with no evidence of effect modification by stroke etiology. ${ }^{23}$ Furthermore, the quality of evidence is also high for the time dependence of benefit of IVT with alteplase in acute ischemic stroke patients with and without the presence of LVO, supporting initiation as quickly as possible. ${ }^{2432}$ The quality of evidence is reported as low in this recommendation specifically for the distinct question as to whether IVT should be withheld in MT-eligible patients arriving at a center without thrombectomy facilities. There are no RCTs to address this specific question and, furthermore, they are unlikely to be performed at this time given the absence of support for this concept from the trials of patients directly admitted to thrombectomy-capable centers.

\section{Expert consensus statement}

For patients with unknown stroke onset, the administration of IVT in a non-thrombectomy-capable center also guarantees faster initiation of reperfusion therapy, but the risk of delayed MT after IVT 


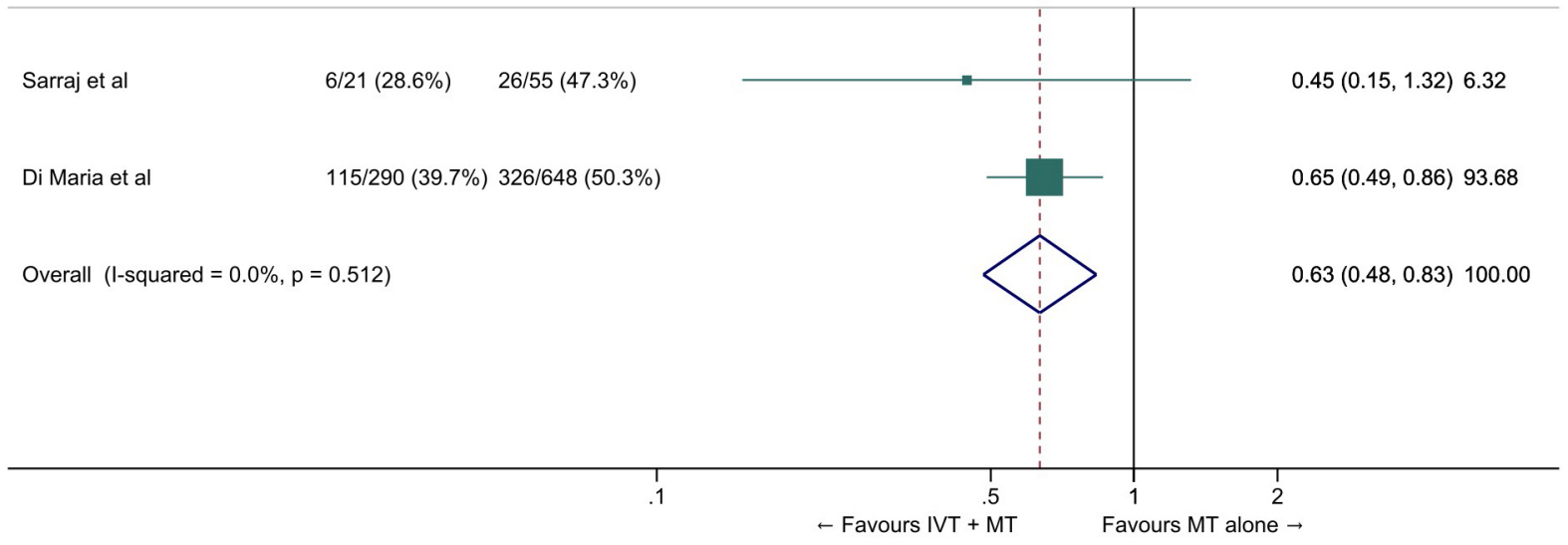

Figure 10 Good outcome (mRS 0-2 at 90 days) in 'drip-and-ship' large vessel occlusion stroke patients treated with MT alone versus IVT plus MT within 4.5 hours of symptom onset (unadjusted pooled OR, random-effects meta-analysis). dMT, direct mechanical thrombectomy (MT alone); IVT, intravenous thrombolysis with alteplase; mRS, modified Rankin Scale; MT, mechanical thrombectomy.

in the drip-and-ship setting is more uncertain given an absence of data. However, following the same rationale as for the 'mothership' patients, the MWG members provide the following expert consensus statement for patients with wake-up stroke admitted to a center without MT facilities. The results of the votes for this statement are provided in online supplemental table 2 . This expert consensus statement supersedes the one provided in the 2021 ESO Guideline on intravenous thrombolysis for patients with wake-up stroke who are eligible for both IVT and $\mathrm{MT}^{23}$

\section{DISCUSSION}

Based on newly available data from six recent RCTs, we strongly recommend that patients with anterior circulation LVO ischemic stroke receive IVT in addition to MT if they are eligible for both treatments. This general recommendation does not exclude individual decision-making under specific conditions, such as for patients at particularly high risk of complications from either treatment. A summary of PICO questions, evidence-based recommendations, and expert consensus statements is provided in table 5 .

The main theoretical benefits from withholding IVT for LVO patients eligible for MT are efficacy (potentially low efficacy of IVT in LVO patients ${ }^{53}$ ), safety (eg, reduction in intracranial hemorrhage rate), logistical (time-saving), and economic savings (cost of alteplase). ${ }^{5455}$ The theoretical advantages of adding IVT include the higher rate of early reperfusion before $\mathrm{MT},{ }^{47} 56$ the potentially higher rate of post-interventional reperfusion with
Study

dMT

IVT + MT

$\mathrm{OR}(95 \% \mathrm{Cl})$

Weight

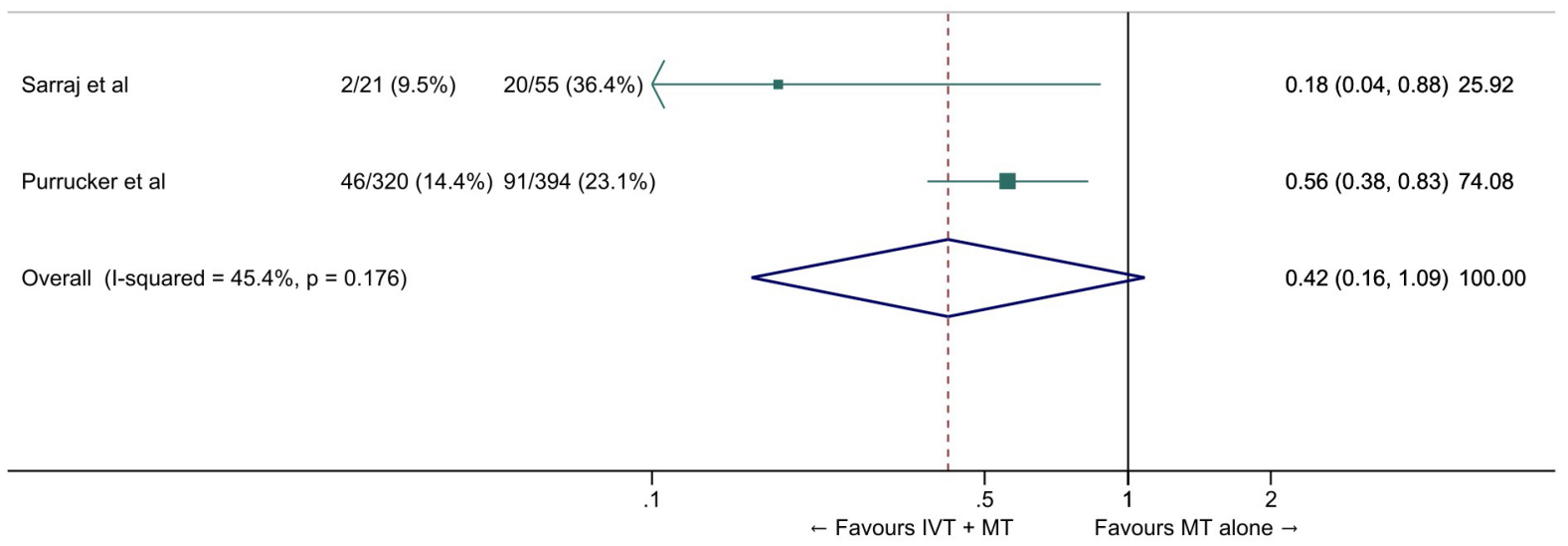

Figure 11 Excellent outcome (mRS 0-1 at 90 days) in 'drip-and-ship' large vessel occlusion stroke patients treated with MT alone versus IVT plus MT within 4.5 hours of symptom onset (unadjusted pooled OR, random-effects meta-analysis). dMT, direct mechanical thrombectomy (MT alone); IVT, intravenous thrombolysis with alteplase; mRS, modified Rankin Scale; MT: mechanical thrombectomy. 


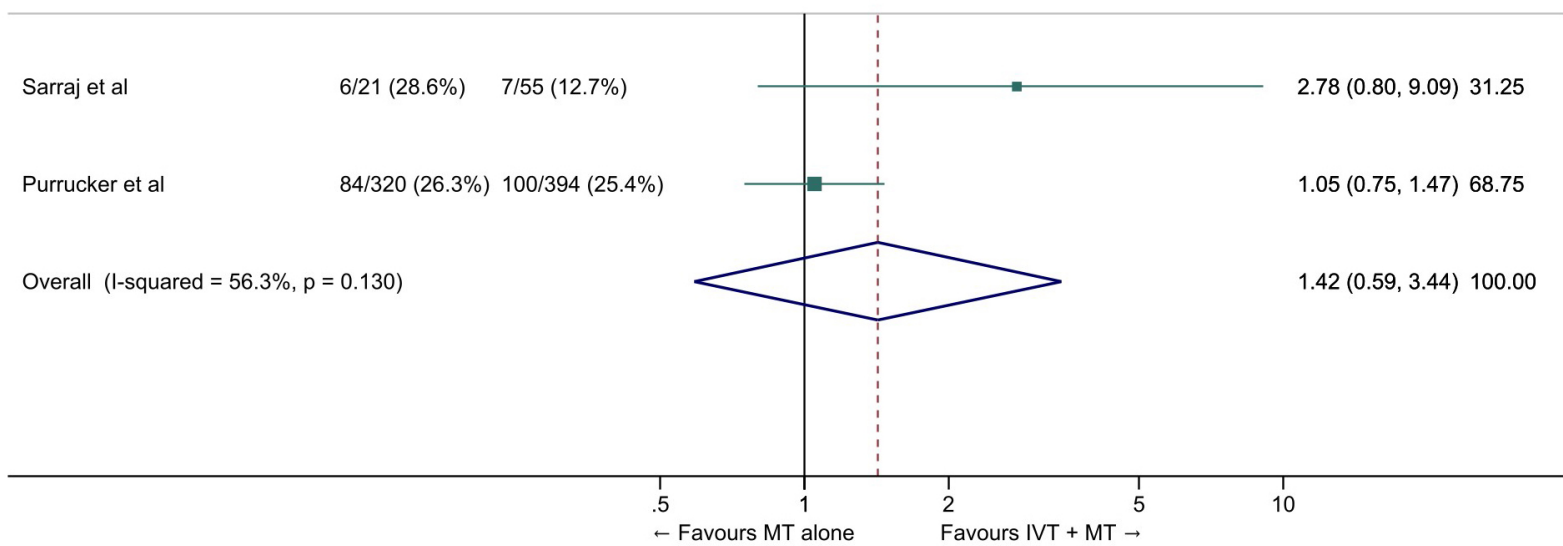

Figure 12 All-cause mortality at 90 days in 'drip-and-ship' large vessel occlusion stroke patients treated with MT alone versus IVT plus MT within 4.5 hours of symptom onset (unadjusted pooled OR, random-effects meta-analysis). dMT, direct mechanical thrombectomy (MT alone), IVT, intravenous thrombolysis with alteplase; MT, mechanical thrombectomy.

fewer recanalization attempts, ${ }^{57}$ potential benefits in patients with failed MT reperfusion attempts, ${ }^{58}$ and consequent economic gains (reduced disability).

The optimal population to assess non-inferiority of MT alone over bridging therapy seemed to be those patients arriving directly in the MT-capable center without having received IVT elsewhere ('mothership' paradigm). The study specific prespecified non-inferiority boundaries were crossed in four of the six RCTs. ${ }^{791112}$ Furthermore, pooled results showed a risk difference of $-1.9 \%$ (95\% CI $-5.9 \%$ to $2.1 \%$ ) between MT alone versus bridging therapy for good functional outcome (mRS 0-2) at 90 days. Therefore, data from over 2300 enrolled patients did not lead to the demonstration that MT alone is non-inferior to bridging therapy according to our pre-specified non-inferiority margin (1.3\%). This margin was based on results of a previous survey aiming to determine the minimal clinically relevant increase in the proportion of patients achieving good functional outcome when considering a novel and safe neuroprotective agent as clinically worthwhile. ${ }^{19}$ Applying this specific minimal clinically important difference as a lower non-inferiority margin for withholding IVT may be considered fairly conservative by some. ${ }^{59}$ However, it should be emphasized that non-inferiority of direct MT would not have been demonstrated even using the maximum clinically important non-inferiority margin (5.0\%)

\section{Evidence-based recommendation}

For patients admitted to a non-thrombectomy-capable center for an acute ischemic stroke ( $\leq 4.5$ hours of symptom onset) with anterior circulation large vessel occlusion and who are eligible for both treatments, we recommend intravenous thrombolysis followed by rapid transfer to a center with thrombectomy facilities over omitting intravenous thrombolysis and transfer to a center with thrombectomy facilities.

Intravenous thrombolysis should not delay the transfer to a center with thrombectomy facilities.

Quality of evidence: Low $\oplus \oplus$

Strength of recommendation: Strong $\uparrow \uparrow$ proposed by our MWG. Only accepting more generous margins, namely $5.9 \%$ (i.e. at least 59 fewer independent outcomes at 90 days among 1000 patients treated with direct MT), would lead to the conclusion of non-inferiority. While a few clinicians might personally consider a $6 \%$ margin as acceptable, one must also keep in mind the effect size of previous positive acute stroke trials. Thus, in the guideline-changing ECASS 3 trial of alteplase versus placebo in the 3-4.5 hour time window, active treatment was associated with a $5 \%$ higher rate of good functional outcomes (i.e. 50 more patients with mRS $0-2$ per 1000 patients treated) ${ }^{60}$ Furthermore, when considering the socioeconomic dimension, a safe neuroprotective would be cost-effective and clinically worthwhile if it improved the outcome of $2-3 \%$ of treated patients. ${ }^{5961}$

The aims of the present document were to conduct a methodologically rigorous appraisal and synthesis of the available evidence and to provide a practical interpretation of the data in the form of pragmatic recommendations, which may help ensure

\section{Expert consensus statement}

For patients admitted to a non-thrombectomy-capable center within 4.5 hours of symptom recognition after wake-up ischemic stroke caused by anterior circulation large vessel occlusion, we suggest intravenous thrombolysis plus mechanical thrombectomy over mechanical thrombectomy alone in selected patients.

The selection criteria for IVT and MT for patients with wake-up stroke are detailed in the corresponding European Guidelines. ${ }^{523}$ Notably, eligibility imaging criteria for IVT include DWI-FLAIR mismatch or perfusion core/penumbra mismatch*.

*Perfusion core/penumbra mismatch:

- Infarct core ${ }^{* *}$ volume $<70 \mathrm{~mL}$

- and critically hypoperfused ${ }^{\dagger}$ volume/infarct core ${ }^{* *}$ volume $>1.2$

- and mismatch volume $>10 \mathrm{~mL}$

${ }^{*}{ }^{*} \mathrm{rCBF}<30 \%$ (CT perfusion) or $\mathrm{ADC}<620 \mu \mathrm{m}^{2} / \mathrm{s}$ (diffusion MRI)

${ }^{\dagger}$ Tmax $>6 \mathrm{~s}$ (perfusion CT or perfusion MRI) 
Table 5 Summary of PICO questions, evidence-based recommendations, and expert consensus statements

\begin{tabular}{l}
\hline Topic/PICO question \\
\hline Mothership \\
PICO 1: For large vessel occlusion acute ischemic stroke \\
( $\leq 4.5$ hours of symptom onset) patients directly admitted \\
to a thrombectomy-capable center and eligible for \\
both treatments, does mechanical thrombectomy alone \\
compared with intravenous thrombolysis plus mechanical \\
thrombectomy lead to: \\
$\quad$ a non-inferior proportion of patients with good \\
outcome (mRS $0-2$ ) at 90 days? \\
non-inferior or better results on other efficacy \\
outcomes (whole range of the mRS; mRS $0-1 ;$ \\
successful reperfusion)? \\
$\quad$ a reduction in the risk of adverse events (mortality at \\
90 days, sICH, any ICH)? \\
a reduction in key time metrics?
\end{tabular}

Drip-and-ship

PICO 2: For large vessel occlusion acute ischemic stroke ( $\leq 4.5$ hours of symptom onset) patients admitted to a non-thrombectomy-capable center and eligible for both treatments, does mechanical thrombectomy alone compared with intravenous thrombolysis plus mechanical thrombectomy lead to:

- a non-inferior proportion of patients with good outcome (mRS $0-2$ ) at 90 days?

- non-inferior or better results on other efficacy outcomes (whole range of the $\mathrm{mRS}$; $\mathrm{mRS} 0-1$; successful reperfusion)?

- a reduction in the risk of adverse events (mortality at Strength of recommendation: Strong $\uparrow \uparrow$ 90 days, $\mathrm{sICH}$, any $\mathrm{ICH})$ ?

- a reduction in key time metrics?
Evidence-based recommendation

For patients directly admitted to a thrombectomy-capable center for an acute ischemic stroke ( $\leq 4.5$ hours of symptom onset) with anterior circulation large vessel occlusion and who are eligible for both treatments, we recommend intravenous thrombolysis plus mechanical thrombectomy over mechanical thrombectomy alone.

Both treatments should be performed as early as possible afte hospital arrival. Mechanical thrombectomy should not prevent the initiation of intravenous thrombolysis and intravenous thrombolysis should not delay mechanical thrombectomy.

Quality of evidence: Moderate $\oplus \oplus \oplus$ Strength of recommendation: Strong $\uparrow$

For patients admitted to a non-thrombectomy-capable center for an acute ischemic stroke ( $\leq 4.5$ hours of symptom onset) with anterior circulation large vessel occlusion and who are eligible for both treatments, we recommend intravenous thrombolysis followed by rapid transfer to a center with thrombectomy facilities over omitting intravenous thrombolysis and transfer to a center with thrombectomy facilities. Intravenous thrombolysis should not delay the transfer to a center with thrombectomy facilities.
Expert consensus statement

For patients directly admitted to a thrombectomy-capable center within 4.5 hours of symptom recognition after wake-up ischemic stroke caused by anterior circulation large vessel occlusion, we suggest intravenous thrombolysis plus mechanical thrombectomy over mechanical thrombectomy alone in selected patients. The selection criteria for IVT and MT for patients with wake-up stroke are detailed in the corresponding European Guidelines. ${ }^{5}$ ${ }^{23}$ Notably, eligibility imaging criteria for IVT include DWI-FLAIR mismatch or perfusion core/penumbra mismatch* .

\author{
*Perfusion core/penumbra mismatch: \\ - Infarct core ${ }^{* *}$ volume $<70 \mathrm{~mL}$ \\ - and critically hypoperfused ${ }^{\dagger}$ volume/infarct core ** \\ volume $>1.2$ \\ - and mismatch volume $>10 \mathrm{~mL}$ \\ ** $\mathrm{rCBF}<30 \%$ (CT perfusion) or ADC $<620 \mu \mathrm{m}^{2} / \mathrm{s}$ (diffusion MRI) \\ ${ }^{\dagger}$ Tmax $>6 \mathrm{~s}$ (perfusion CT or perfusion MRI)
}

For patients admitted to a non-thrombectomy-capable center within 4.5 hours of symptom recognition after wake-up ischemic stroke caused by anterior circulation large vessel occlusion, we suggest intravenous thrombolysis plus mechanical thrombectomy over mechanical thrombectomy alone in selected patients.

The selected criteria for IVT and MT for patients with wake-up stroke are detailed in the corresponding European guidelines. ${ }^{5}$

${ }^{23}$ Notably, eligibility imaging criteria for IVT include DWI-FLAIR mismatch or perfusion core/penumbra mismatch*.

*Perfusion core/penumbra mismatch:

- Infarct core** volume $<70 \mathrm{~mL}$

- and critically hypoperfused ${ }^{\dagger}$ volume/infarct core ${ }^{* *}$ volume $>1.2$

- and mismatch volume $>10 \mathrm{~mL}$

${ }^{* *} \mathrm{rCBF}<30 \%$ (CT perfusion) or $\mathrm{ADC}<620 \mu \mathrm{m}^{2} / \mathrm{s}$ (diffusion MRI) ${ }^{\dagger}$ Tmax $>6 \mathrm{~s}$ (perfusion CT or perfusion MRI)

\begin{abstract}
$\mathrm{ADC}$, apparent diffusion coefficient ; CT, computed tomography; DWI-FLAIR, diffusion weighted imaging-fluid attenuated inversion recovery; ICH, intracranial hemorrhage; IVT, intravenous thrombolysis with alteplase; MRI, magnetic resonance imaging; mRS, modified Rankin Scale; MT, mechanical thrombectomy; PICO, Population, Intervention, Comparator, Outcome; rCBF, relative cerebral blood flow; sICH, symptomatic intracranial hemorrhage.
\end{abstract}

equity in access to care in different locations and organizational settings. However, some important points need to be considered for the practical application of our recommendations. First, the results of the available RCTs are strictly applicable to 'mothership' patients only and must not be extrapolated to patients who receive IVT in other centers ('drip-and-ship') or are treated by interventionalists from other centers ('drip-and-drive' or 'dripand-fly') based mainly on the different times of IVT exposure and interval between start of IVT and MT and thus potentially different effectiveness. The data based on currently available RCTs have no bearing on these 'non-mothership' patients and should not be used to guide their management and related logistics. Based on systematic reviews and meta-analyses of the available observational data, we strongly recommend IVT in drip-andship patients. Furthermore, short door-to-IVT and door-to-groin times remain critical in optimizing the chain of care of patients with acute LVO. Second, the results of the RCTs are only valid for patients who can be treated with IVT within 4.5 hours after symptom onset. Consequently, we could only address the question of whether IVT should be administered in selected patients with unknown time of onset by means of expert consensus. It is noteworthy that, in the setting of late time window anterior circulation LVO stroke, strong evidence exists on the efficacy of MT, ${ }^{62}{ }^{63}$ whereas the available evidence for IVT is limited to patients who did not undergo MT. ${ }^{36} 38$ Third, it is important to consider that the RCTs only addressed the question of direct MT versus bridging therapy in anterior circulation occlusion strokes. However, because the level of evidence for MT in basilar artery occlusion is lower than for anterior circulation occlusion, ${ }^{64-66}$ with no evidence of heterogeneity for the strong treatment effect of IVT, ${ }^{67}$ the MWG members deemed this subgroup as lying outside the scope of this expedited recommendation document. Fourth, not only the inclusion criteria but also the population actually enrolled in the RCTs should be considered (table 2). In this regard, even though patients with a very low NIHSS score or a large infarct volume could be enrolled in three of the trials, ${ }^{6} 89$ few of such patients were actually randomized and the generalizability of the available results to these populations is therefore uncertain. Fifth, the trials included in this present analysis provide information regarding IVT with alteplase only; reliable evidence for other fibrinolytic agents, such as tenecteplase, or for a combination of antithrombotics, is lacking. Sixth, at this time none of the subgroup analyses published so far has disclosed a significant modification of the treatment effect in specific situations. Likewise, the rate of sICH ranged from $4.8 \%$ to $6.8 \%$ in patients randomized to bridging therapy in the five RCTs where the full dose of alteplase was delivered with no evidence of heterogeneity across predefined subgroups. Future individual patient-level meta-analyses of all RCTs might disclose subgroups in whom direct MT is superior to IVT plus MT, which could lead to more personalized treatment strategies. Finally, all the trials were conducted using currently available MT devices; as new and more effective devices may lead to faster and more complete reperfusion, updating of the present recommendations may be required in the future.

\section{Author affiliations}

${ }^{1}$ Department of Neurology, GHU Paris Psychiatrie et Neurosciences, Université de Paris, INSERM U1266, FHU NeuroVasc, Paris, France

${ }^{2}$ Second Department of Neurology, Attikon University Hospital, School of Medicine, National and Kapodistrian University of Athens, Athens, Greece 
${ }^{3}$ Department of Neurology, University of Tennessee Health Science Center, Memphis, Tennessee, USA

${ }^{4}$ Klinik und Hochschulambulanz für Neurologie, Campus Benjamin Franklin, Charité Universitätsmedizin Berlin \& Center for Stroke Research Berlin, Berlin, Germany ${ }^{5}$ Department of Neurosurgery, Radboud University Medical Center, Nijmegen, The Netherlands

${ }^{6}$ Department of Interventional Neuroradiology, Royal London Hospital, Barts NHS Trust, London, UK

${ }^{7}$ Neurology and Stroke Center, University Hospital of Basel, University of Basel, Basel, Switzerland

${ }^{8}$ Department of Neurosciences and Mental Health (Neurology), Hospital Santa Maria-CHLN, Faculdade de Medicina, Universidade de Lisboa, Lisboa, Portugal ${ }^{9}$ Department of Neurology, University of Cincinnati, Cincinnati, Ohio, USA ${ }^{10}$ Department of Interventional Neuroradiology, Rothschild Foundation Hospital, Paris, France

${ }^{11}$ Stroke Unit, Lariboisière Hospital AP-HP-Nord, FHU NeuroVasc, Université de Paris, Paris, France

${ }^{12}$ Stroke Unit, Department of Neurology, Germans Trias i Pujol Hospital, Barcelona, Spain

${ }^{13}$ Departments of Neurology and Neurogeriatrics, Johannes Wesling Medical Center Minden, University hospitals of the Ruhr-University of Bochum, Bochum, Germany

${ }^{14}$ Department of Neurology, Helsinki University Hospital and University of Helsinki, Helsinki, Finland

${ }^{15}$ Hospital Policlinico Umberto I, Department of Human Neurosciences, Sapienza University of Rome, Rome, Italy

${ }^{16}$ Translational and Clinical Research Institute, Newcastle University, Newcastle upon Tyne Hospitals NHS Foundation Trust, Newcastle upon Tyne, UK

${ }^{17}$ Centre for Clinical Brain Sciences, University of Edinburgh, Edinburgh, UK

${ }^{18}$ IRCCS Istituto delle Scienze Neurologiche di Bologna, Department of Neurology and Stroke Center, Maggiore Hospital, Bologna, Italy

${ }^{19}$ Department of Radiology and Nuclear Medicine, Maastricht University Medical Center and Cardiovascular Research Institute Maastricht (CARIM), Maastricht, The Netherlands

${ }^{20}$ Department of Diagnostic and Interventional Neuroradiology, University Medical Center Hamburg-Eppendorf, Hamburg, Germany

\section{Twitter Jens Fiehler @Fie0815}

Acknowledgements We thank Ale Algra, Eric Vicaut, and Jean-Claude Baron for useful discussions.

Contributors GTc, JF and GTs wrote the first draft of the manuscript. GTc conducted the statistical analysis. All authors reviewed and edited the manuscript and approved the final version of the manuscript.

Funding The authors have not declared a specific grant for this research from any funding agency in the public, commercial or not-for-profit sectors.

Competing interests All disclosures are provided in Supplemental Table 1.

Patient consent for publication Not applicable.

Ethics approval This study does not involve human participants.

Provenance and peer review Not commissioned; externally peer reviewed.

Supplemental material This content has been supplied by the author(s). It has not been vetted by BMJ Publishing Group Limited (BMJ) and may not have been peer-reviewed. Any opinions or recommendations discussed are solely those of the author(s) and are not endorsed by BMJ. BMJ disclaims all liability and responsibility arising from any reliance placed on the content. Where the content includes any translated material, BMJ does not warrant the accuracy and reliability of the translations (including but not limited to local regulations, clinical guidelines, terminology, drug names and drug dosages), and is not responsible for any error and/or omissions arising from translation and adaptation or otherwise.

\section{ORCID iDs}

Guillaume Turc http://orcid.org/0000-0001-5059-4095

Georgios Tsivgoulis http://orcid.org/0000-0002-0640-3797

Hieronymus Boogaarts http://orcid.org/0000-0001-5855-2447

Pervinder Bhogal http://orcid.org/0000-0002-5514-5237

Gian Marco De Marchis http://orcid.org/0000-0002-0342-9780

Ana Catarina Fonseca http://orcid.org/0000-0001-6913-5526

Pooja Khatri http://orcid.org/0000-0002-7344-8266

Mikaël Mazighi http://orcid.org/000-0003-0911-8999

Philip White http://orcid.org/0000-0001-6007-6013

William Whiteley http://orcid.org/0000-0002-4816-8991

Andrea Zini http://orcid.org/0000-0003-1486-4507

Wim van Zwam http://orcid.org/0000-0003-1631-7056

Jens Fiehler http://orcid.org/0000-0001-8533-7478

\section{REFERENCES}

1 National Institute of Neurological Disorders and Stroke rt-PA Stroke Study Group. Tissue plasminogen activator for acute ischemic stroke. N Engl J Med 1995;333:1581-7.

2 Hacke W, Kaste M, Fieschi C, et al. Randomised double-blind placebo-controlled trial of thrombolytic therapy with intravenous alteplase in acute ischaemic stroke (ECASS II). Second European-Australasian Acute Stroke Study Investigators. Lancet 1998;352:1245-51.

3 Thomalla G, Simonsen CZ, Boutitie F, et al. MRI-guided thrombolysis for stroke with unknown time of onset. N Engl J Med 2018;379:611-22.

$4 \mathrm{Ma} \mathrm{H}$, Campbell BCV, Parsons MW, et al. Thrombolysis guided by perfusion imaging up to 9 hours after onset of stroke. N Engl J Med 2019;380:1795-803.

5 Turc G, Bhogal P, Fischer U, et al. European Stroke Organisation (ESO) - European Society for Minimally Invasive Neurological Therapy (ESMINT) guidelines on mechanical thrombectomy in acute ischaemic stroke. Endorsed by Stroke Alliance for Europe (SAFE). Eur Stroke J 2019;4:6-12.

6 Yang P, Zhang Y, Zhang L, et al. Endovascular thrombectomy with or without intravenous alteplase in acute stroke. N Eng/ J Med 2020;382:1981-93.

7 Suzuki K, Matsumaru Y, Takeuchi M, et al. Effect of mechanical thrombectomy without vs with intravenous thrombolysis on functional outcome among patients with acute ischemic stroke: the SKIP randomized clinical trial. JAMA 2021;325:244-53.

8 Zi W, Qiu Z, Li F, et al. Effect of endovascular treatment alone vs intravenous alteplase plus endovascular treatment on functional independence in patients with acute ischemic stroke: the DEVT randomized clinical trial. JAMA 2021;325:234-43.

9 LeCouffe NE, Kappelhof M, Treurniet KM, et al. A randomized trial of intravenous alteplase before endovascular treatment for stroke. N Engl J Med 2021;385:1833-44.

10 Katsanos AH, Turc G, Psychogios M, et al. Utility of intravenous alteplase prior to endovascular stroke treatment: a systematic review and meta-analysis of RCTs. Neurology 2021;97:e777-84.

11 Fischer U, Gralla J, SD I. Solitaire with the intention for thrombectomy plus intravenous t-PA versus direct Solitaire stent retriever thrombectomy in acute anterior circulation stroke (SWIFT DIRECT). Eur Stroke J 2021;6:5.

12 Mitchell PJ, Yan B. A randomized controlled trial of direct endovascular clot retrieval versus standard bridging thrombolysis with endovascular clot retrieval within 4.5 hours of stroke onset. Int I Stroke 2021;16:171-97.

13 Ntaios G, Bornstein NM, Caso V, et al. The European Stroke Organisation guidelines: a standard operating procedure. Int I Stroke 2015;10 Suppl A100:10 Suppl A100:128-35

14 Steiner T, Dichgans M, Norrving B, et al. European Stroke Organisation (ESO) standard operating procedure for the preparation and publishing of guidelines. Eur Stroke $J$ 2021:6:CXXII-CXXXIV.

15 Guyatt GH, Oxman AD, Schünemann HJ, et al. GRADE guidelines: a new series of articles in the Journal of Clinical Epidemiology. I Clin Epidemiol 2011;64:380-2.

16 Saver JL, Chaisinanunkul N, Campbell BCV, et al. Standardized Nomenclature for modified Rankin Scale global disability outcomes: consensus recommendations from stroke therapy academic industry roundtable XI. Stroke 2021;52:3054-62.

17 Wang Y, Wu X, Zhu C, et al. Bridging thrombolysis achieved better outcomes than direct thrombectomy after large vessel occlusion: an updated meta-analysis. Stroke 2021;52:356-65

18 Sterne JAC, Savović J, Page MJ, et al. RoB 2: a revised tool for assessing risk of bias in randomised trials. BMJ 2019;366:14898.

19 Cranston JS, Kaplan BD, Saver JL. Minimal clinically important difference for safe and simple novel acute ischemic stroke therapies. Stroke 2017;48:2946-51.

20 DerSimonian R, Laird N. Meta-analysis in clinical trials. Control Clin Trials 1986;7:177-88.

21 Pan Y, Chen Q, Zhao X, et al. Cost-effectiveness of thrombolysis within 4.5 hours of acute ischemic stroke in China. PLoS One 2014;9:e110525.

22 Nogueira RG, Tsivgoulis G. Large vessel occlusion strokes after the DIRECT-MT and SKIP trials: is the alteplase syringe half empty or half full? Stroke 2020;51:3182-6.

23 Berge E, Whiteley W, Audebert $\mathrm{H}$, et al. European Stroke Organisation (ESO) guidelines on intravenous thrombolysis for acute ischaemic stroke. Eur Stroke J 2021;6:I-LXII.

24 Powers WJ, Rabinstein AA, Ackerson T, et al. Guidelines for the early management of patients with acute ischemic stroke: 2019 update to the 2018 guidelines for the early management of acute ischemic stroke: a guideline for healthcare professionals from the American Heart Association/American Stroke Association. Stroke 2019;50:e344-418.

25 Jauch EC, Saver JL, Adams HP, et al. Guidelines for the early management of patients with acute ischemic stroke: a guideline for healthcare professionals from the American Heart Association/American Stroke Association. Stroke 2013;44:870-947.

26 Katsanos AH, Malhotra K, Goyal N, et al. Intravenous thrombolysis prior to mechanical thrombectomy in large vessel occlusions. Ann Neurol 2019;86:395-406.

27 Du H, Lei H, Ambler G, et al. Intravenous thrombolysis before mechanical thrombectomy for acute ischemic stroke: a meta-analysis. J Am Heart Assoc 2021; 10:e022303.

28 Jang KM, Choi HH, Jang M-jin, et al. Direct endovascular thrombectomy alone vs. bridging thrombolysis for patients with acute ischemic stroke. Clin Neuroradiol 2021;10. 
29 Zhang J, Chen S, Shi S, et al. Direct endovascular treatment versus bridging therapy in patients with acute ischemic stroke eligible for intravenous thrombolysis: systematic review and meta-analysis. J Neurointerv Surg 2021. doi:10.1136/ neurintsurg-2021-017928. [Epub ahead of print: 04 Aug 2021].

30 Li H, Yang S, Zhong Y, et al. Mechanical thrombectomy with or without intravenous thrombolysis in acute ischemic stroke: a meta-analysis for randomized controlled trials. Eur Neurol 2021:1-10.

31 Requena M, Olivé-Gadea M, Muchada M, et al. Direct to angiography suite without stopping for computed tomography imaging for patients with acute stroke: a randomized clinical trial. JAMA Neurol 2021;78:1099-107.

32 Fischer U, Kaesmacher J, S Plattner P, et al. SWIFT DIRECT: Solitaire ${ }^{T M}$ with the intention for thrombectomy plus intravenous t-PA versus direct Solitaire ${ }^{T M}$ stentretriever thrombectomy in acute anterior circulation stroke: methodology of a randomized, controlled, multicentre study. Int J Stroke 2021:174749302110487.

33 Treurniet KM, LeCouffe NE, Kappelhof M, et al. Mr CLEAN-NO IV: intravenous treatment followed by endovascular treatment versus direct endovascular treatment for acute ischemic stroke caused by a proximal intracranial occlusion-study protocol for a randomized clinical trial. Trials 2021;22:141.

34 Ringleb P, Bendszus M, Bluhmki E, et al. Extending the time window for intravenous thrombolysis in acute ischemic stroke using magnetic resonance imaging-based patient selection. Int J Stroke 2019;14:483-90.

35 Davis SM, Donnan GA, Parsons MW, et al. Effects of alteplase beyond $3 \mathrm{~h}$ after stroke in the Echoplanar Imaging Thrombolytic Evaluation Trial (EPITHET): a placebocontrolled randomised trial. Lancet Neurol 2008;7:299-309.

36 Campbell BCV, Ma H, Ringleb PA, et al. Extending thrombolysis to 4.5-9 h and wake-up stroke using perfusion imaging: a systematic review and meta-analysis of individual patient data. Lancet 2019;394:139-47.

37 Koga $\mathrm{M}$, Yamamoto $\mathrm{H}$, Inoue $\mathrm{M}$, et al. Thrombolysis with alteplase at $0.6 \mathrm{mg} /$ $\mathrm{kg}$ for stroke with unknown time of onset: a randomized controlled trial. Stroke 2020;51:1530-8.

38 Thomalla G, Boutitie F, Ma H, et al. Intravenous alteplase for stroke with unknown time of onset guided by advanced imaging: systematic review and meta-analysis of individual patient data. Lancet 2020;396:1574-84.

39 Roaldsen MB, Lindekleiv $\mathrm{H}$, Eltoft A, et al. Tenecteplase in wake-up ischemic stroke trial: protocol for a randomized-controlled trial. Int I Stroke 2021;16:990-994.

40 Tsivgoulis G, Saqqur M, Sharma VK, et al. Timing of recanalization and functional recovery in acute ischemic stroke. J Stroke 2020;22:130-40.

41 Romoli M, Paciaroni M, Tsivgoulis G, et al. Mothership versus drip-and-ship model for mechanical thrombectomy in acute stroke: a systematic review and meta-analysis for clinical and radiological outcomes. J Stroke 2020;22:317-23.

42 Sarraj A, Savitz S, Pujara D, et al. Endovascular thrombectomy for acute ischemic strokes: current US access paradigms and optimization methodology. Stroke 2020;51:1207-17.

43 Aguiar de Sousa D, von Martial R, Abilleira S, et al. Access to and delivery of acute ischaemic stroke treatments: a survey of national scientific societies and stroke experts in 44 European countries. Eur Stroke J 2019;4:13-28.

44 Southerland AM, Johnston KC, Molina CA, et al. Suspected large vessel occlusion: should emergency medical services transport to the nearest primary stroke center or bypass to a comprehensive stroke center with endovascular capabilities? Stroke 2016;47:1965-7.

45 Milne MSW, Holodinsky JK, Hill MD, et al. Drip 'n ship versus mothership for endovascular treatment: modeling the best transportation options for optimal outcomes. Stroke 2017;48:791-4.

46 Purrucker JC, Heyse M, Nagel S, et al. Efficacy and safety of bridging thrombolysis initiated before transfer in a drip-and-ship stroke service. Stroke Vasc Neurol 2021. doi:10.1136/svn-2021-001024. [Epub ahead of print: 26 Jul 2021].

47 Seners $P$, Turc $G$, Naggara 0 , et al. Post-thrombolysis recanalization in stroke referrals for thrombectomy: incidence, predictors, and prediction scores. Stroke 2018;49:2975-82.
48 Mueller L, Pult F, Meisterernst J, et al. Impact of intravenous thrombolysis on recanalization rates in patients with stroke treated with bridging therapy. Eur J Neurol 2017;24:1016-21

49 Sarraj A, Grotta J, Albers GW, et al. Clinical and neuroimaging outcomes of direct thrombectomy vs bridging therapy in large vessel occlusion: analysis of the SELECT cohort study. Neurology 2021;96:e2839-53.

50 Di Maria F, Mazighi M, Kyheng M, et al. Intravenous thrombolysis prior to mechanical thrombectomy in acute ischemic stroke: silver bullet or useless bystander? I Stroke 2018;20:385-93.

51 Pérez de la Ossa N, Abilleira S, Jiménez X. Transfer to the local stroke center versus direct transfer to endovascular center of acute stroke patients with suspected large vessel occlusion in the Catalan Territory (RACECAT). European Stroke Organisation Conference, 2020.

52 McTaggart RA, Moldovan K, Oliver LA, et al. Door-in-door-out time at primary stroke centers may predict outcome for emergent large vessel occlusion patients. Stroke 2018;49:2969-74.

53 Seners $P$, Turc $G$, Maïer $B$, et al. Incidence and predictors of early recanalization after intravenous thrombolysis: a systematic review and meta-analysis. Stroke 2016;47:2409-12.

54 Fischer U, Kaesmacher J, Mendes Pereira V, et al. Direct mechanical thrombectomy versus combined intravenous and mechanical thrombectomy in large-artery anterior circulation stroke: a topical review. Stroke 2017;48:2912-8.

55 Ospel JM, McDonough R, Kunz WG, et al. Is concurrent intravenous alteplase in patients undergoing endovascular treatment for large vessel occlusion stroke cost-effective even if the cost of alteplase is only US\$1? J Neurointerv Surg 2021. doi:10.1136/neurintsurg-2021-017817. [Epub ahead of print: 29 Jun 2021].

56 Tsivgoulis G, Katsanos AH, Schellinger PD, et al. Successful reperfusion with intravenous thrombolysis preceding mechanical thrombectomy in large-vessel occlusions. Stroke 2018;49:232-5.

57 Fischer U, Kaesmacher J, Molina CA, et al. Primary thrombectomy in tPA (tissue-type plasminogen activator) eligible stroke patients with proximal intracranial occlusions. Stroke 2018;49:265-9.

58 Rozes C, Maier B, Gory B, et al. Influence of prior intravenous thrombolysis on outcome after failed mechanical thrombectomy: ETIS registry analysis. J Neurointerv Surg 2021. doi:10.1136/neurintsurg-2021-017867. [Epub ahead of print: 19 Aug 2021].

59 Lin C-H, Saver JL, Ovbiagele B, et al. Endovascular thrombectomy without versus with intravenous thrombolysis in acute ischemic stroke: a non-inferiority metaanalysis of randomized clinical trials. J Neurointerv Surg 2021. doi:10.1136/ neurintsurg-2021-017667. [Epub ahead of print: 15 Jul 2021].

60 Hacke W, Kaste M, Bluhmki E, et al. Thrombolysis with alteplase 3 to 4.5 hours after acute ischemic stroke. N Engl J Med 2008;359:1317-29.

61 Samsa GP, Matchar DB. Have randomized controlled trials of neuroprotective drugs been underpowered? An illustration of three statistical principles. Stroke 2001;32:669-74.

62 Albers GW, Marks MP, Kemp S, et al. Thrombectomy for stroke at 6 to 16 hours with selection by perfusion imaging. N Eng/ J Med 2018;378:708-18.

63 Nogueira RG, Jadhav AP, Haussen DC, et al. Thrombectomy 6 to 24 hours after stroke with a mismatch between deficit and infarct. $N$ Engl J Med 2018;378:11-21.

64 Langezaal LCM, van der Hoeven EJRJ, Mont'Alverne FJA, et al. Endovascular therapy for stroke due to basilar-artery occlusion. N Engl J Med 2021;384:1910-20.

65 Liu X, Dai Q, Ye R, et al. Endovascular treatment versus standard medical treatment for vertebrobasilar artery occlusion (BEST): an open-label, randomised controlled trial. Lancet Neurol 2020;19:115-22.

66 Schonewille WJ, Wijman CAC, Michel P, et al. Treatment and outcomes of acute basilar artery occlusion in the Basilar Artery International Cooperation Study (BASICS): a prospective registry study. Lancet Neurol 2009;8:724-30.

67 Dorňák T, Král M, Hazlinger M, et al. Posterior vs. anterior circulation infarction: demography, outcomes, and frequency of hemorrhage after thrombolysis. Int J Stroke 2015;10:1224-8. 